\title{
Modeling Competitive Reaction Mechanisms of Peroxynitrite Oxidation of Guanine
}

\author{
Ning Liu, Fuqiang Ban and Russell J. Boyd* \\ Contribution from the Department of Chemistry, Dalhousie University, Halifax, NS, \\ Canada B3H $4 J 3$
}

Supporting Information 
G. ( - H)

$1 \backslash 1 \backslash G \mid N C-N 3 \backslash F 0 p t \backslash U B 3 L Y P \backslash 6-31 G(d, p) \backslash C 5 H 4 N 501(2) \backslash L I U \backslash 07-0 c t-2004 \backslash 0 \backslash \backslash \# B 3 L$ $Y P / 6-31 G * *$ OPT SCRF =(DIPOLE, SOLVENT=WATER) ||PCM GUO||0,2|C, 0.515084841 $4,-0.8726480412,0.0000956533 \backslash \mathrm{C}, 0.798216322,0.5292296349,0.0000717495 \backslash \mathrm{C}$ - $0.3548200947,1.4526866385,0.00029925331 N^{\prime}, 1.5964667136,0.8373577216$, $0.0000729586 \backslash C_{1}-1.6848153344,-0.4803465151,0.0000667214 \backslash N,-0.661356340$ $8,-1.439214143,0.00013850481 N, 1.7575939727,-1.4798270084,-0.0000412352$ IC, 2. $6755938413,-0.4703395388,-0.000067415 \mid N, 2.1352242737,0.7498703491$ , - $0.0001138961 \backslash 0,-0.2204212544,2.6793982937,-0.0002646617 \backslash \mathrm{H}, 3.73851358$ $63,-0.6715945518,-0.00017207831 \mathrm{~N},-2.9059766601,-1.0549654383,-0.000078$ $35991 \mathrm{H},-3.7256269518,-0.4715266492,-0.00016928561 \mathrm{H},-2.9836298931,-2.06$ $00189396,-0.00009742681 \mathrm{H}, 1.9354261174,-2.4760896455,-0.00008549521$ V Ver si on $=x 86$. Li nux-G03RevB. $05 \backslash S t$ at $e=2$. A $\backslash$ Di el ect r i $C=78.39 \backslash A 0=4.22 \backslash H F=-541.9$ $189863 \backslash S 2=0.766085 \backslash S 2-1=0 . \mid S 2 A=0.750201 \backslash R M S D=8.360 e-09 \backslash R M S F=1.383 e-04 \backslash$ Di pole $=0.4691399,-3.9317292,0.0002129 \mid P G=C 01[X(C 5 H 4 N 501)] \backslash 1 @$

$\mathrm{G}^{+}$

$1 \backslash 1 \backslash G \mid N C-N 3 \backslash F O p t \backslash U B 3 L Y P \backslash 6-31 G(d, p) \backslash C 5 H 5 N 501(1+, 2) \backslash L I U \backslash 07-0 c t-2004 \backslash 0 \backslash \backslash \#$

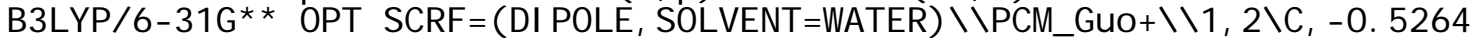
$498466,-0.8748262213,0.00000074 \backslash C,-0.8554924668,0.521728964,-0.0000111$ $273 \backslash C, 0.2438193108,1.4842520804,-0.0000224985 \backslash N, 1.5048509677,0.8106004$ $939,0.0000001508 \backslash C, 1.6942561036,-0.5469797229,0.0000109087 \backslash N, 0.6570103$ $817,-1.4320722974,0.0000069599 \mid N,-1.7478944742,-1.5095645682,-0.000020$ $9016 \backslash \mathrm{C},-2.6933121299,-0.5318084527,0.0000020615 \backslash N,-2.1861663744,0.7097$ $161811,0.0000004207 \backslash 0,0.1814901217,2.69476426,-0.0000023764 \backslash \mathrm{H},-3.75034$ $66169,-0.760469454,0.0000009569 \backslash N, 2.9072928064,-1.0675347211,0.0000272$ $369 \mid \mathrm{H}, 3.7637650122,-0.5252634336,0.00003341021 \mathrm{H}, 2.995815099,-2.0820793$ $011,0.0000351504 \backslash H,-1.8918964883,-2.5154012071,-0.0000346666 \backslash \mathrm{H}, 2.30816$ $30449,1.432883812,0.0000065877$ । Versi on=x86- Li nux-G03RevB.05\St at e=2-A $\backslash \mathrm{Di}$ electric $=78.39 \backslash \mathrm{AO}=3.921 \mathrm{HF}=-542.3098086 \mid S 2=0.762761 \backslash \mathrm{S} 2-1=0.1 \mathrm{~S} 2 \mathrm{~A}=0.75$ $0134 \backslash$ RMSD $=5.241 \mathrm{e}-09 \backslash \mathrm{RMSF}=3.189 \mathrm{e}-05 \backslash \mathrm{Di}$ pol e $=2.3403744,-3.2575954,0.00000$ $6 \backslash P G=C O 1[X(C 5 H 5 N 501)] \backslash \backslash @$

$1 \backslash 1 \backslash G \mid N C-N 5 \backslash F O P t \backslash R B 3 L Y P \backslash 6-31 G(d, p) \backslash C 5 H 5 N 603(1+) \backslash L I U \backslash 07-0 C t-2004 \backslash 0 \backslash \backslash \# B 3$ $L Y P / 6-31 G * *$ OPT SCRF=( DI POLE, SOLVENT =WATER) $\mid \backslash P C M$ a $\mid \backslash 1,1 \backslash C,-0.352037569$ $6,-0.8271375351,-0.2086129449 \mid C_{1}-0.1809598434,0 . \overline{6} 400412885,-0.19683652$ $43 \backslash \mathrm{C},-1.3865979954,1.4706901994,0.1117582394 \backslash \mathrm{N},-2.5442068856,0.6723256$ $378,0.1962712063 \backslash C_{1}-2.5911738058,-0.7055598952,0.0796057264 \backslash N,-1.45730$ $2286,-1.4745611388,-0.0726417897 \backslash N, 0.8997863138,-1.3062774813,-0.43816$ $00276 \backslash \mathrm{C}, 1.7670114822,-0.2036411384,-0.7166191964 \backslash \mathrm{N}, 1.0125896612,1.0223$ $248077,-0.4690907741 \backslash 0,-1.3783744361,2.6650693918,0.2700292748 \backslash \mathrm{H}, 2.199$ $1120558,-0.2066318469,-1.7205293874 \backslash N,-3.7344952497,-1.3354767901,0.11$ $484432131 \mathrm{H},-4.6473248172,-0.8847920591,0.1930904106 \mid \mathrm{H},-3.7462210641,-2$ $.3531371471,0.0233930548 \backslash H_{-}-3.4118580359,1.1902011929,0.3413341535 \backslash H_{1} 1$ $.085018917,-2.2750116559,-0.6731122999 \backslash \mathrm{N}, 3.0272621155,-0.1277456528,0$. $2144235316|0,4.0061901154,0.4163836587,-0.2903799295| 0,2.9419822772,-0$ $.5653420103,1.3454165288$ । Versi on =x86- Li nux-G03RevB.05\St at e=1-AlDi ele ctric $=78.39 \backslash A 0=4.12 \backslash \mathrm{HF}=-747.4201602 \backslash \mathrm{RMSD}=5.278 \mathrm{e}-09 \backslash \mathrm{RMSF}=1.765 \mathrm{e}-05 \backslash \mathrm{Di} \mathrm{po}$ $|e=-6.9120033,-3.0469698,-0.9159339| P G=C 01[X(C 5 H 5 N 603)] \mid \backslash @$

\section{b}

$1 \backslash 1 \backslash G \mid N C-N 07 \backslash F O p t \backslash R B 3 L Y P \backslash 6-31 G(d, p) \backslash C 5 H 5 N 603(1+) \backslash L I U N \backslash 16-D e C-2004 \backslash 0 \backslash \backslash \#$ $B 3 L Y P / 6-31 G * *$ OPT $S C R F=(D \mid P O L E, S O L V E N T=W A T E R) \backslash|P C M \quad b \backslash| 1,1 \backslash C, 0.12181537$ $09,1.1747711874,0.10445650921 \mathrm{C}, 0.6820288269,-0.1822778251,-0.284051314$ $4 \backslash C,-0.3925942403,-1.0330821645,-0.9710052077 \backslash N,-1.7000108752,-0.59857$ $53113,-0.6832092555 \backslash \mathrm{C},-2.0287642358,0.5228136565,0.0457495434 \backslash \mathrm{N},-1.103$ $005027,1.4638130646,0.3826739721 \backslash N, 1.1570650502,2.0279801219,-0.067125$ $7548 \backslash C, 2.1571945442,1.3196362297,-0.7551430493 \backslash N, 1.9234053601,0.069305$ $8623,-0.951638569910,-0.1608393204,-2.0015302024,-1.64363992781 \mathrm{H}, 3.043$ 
$3347402,1.8435877535,-1.0947800917 \backslash N,-3.2790498991,0.7717666081,0.3562$ $546076 / \mathrm{H},-3.5007315948,1.6607743218,0.80198685261 \mathrm{H},-4.0594900202,0.172$ $7152673,0.1075097344 \backslash \mathrm{H},-2.4356956421,-1.1659860076,-1.0972513407 \backslash \mathrm{H}, 1.0$ $831683711,3.0387816812,0.0164640616 \backslash \mathrm{N}, 1.0148925811,-0.9707082132,1.090$ $9228246 \mid 0,2.1692127386,-0.9608455012,1.4565984861 \backslash 0,0.0589081094,-1.50$

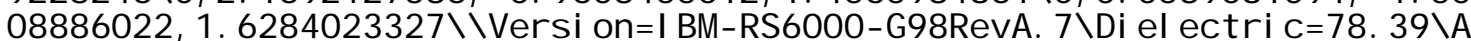
$0=4.17 \backslash \mathrm{HF}=-747.3989034 \backslash \mathrm{RMSD}=7.595 \mathrm{e}-09 \backslash \mathrm{RMSF}=1.758 \mathrm{e}-05 \backslash \mathrm{Di} \mathrm{pole}=-3.8683956$ , 3. $5166742,-0.4050327 \backslash P G=C 01[X(C 5 H 5 N 603)] \backslash 1 @$

C

$1 \backslash 1 \backslash G|N C-N 3 \backslash F O p t \backslash R B 3 L Y P \backslash 6-31 G(d, p) \backslash C 5 H 7 N 604(1+) \backslash L| U \backslash 07-0 c t-2004 \backslash 0 \backslash \backslash \# B 3$ LYP/ 6-31G** OPT SCRF=(DI POLE, SOLVENT =WATER) \|PCM C \\1,1\C,-0.063949431 $7,-1.288104739,-0.0661310915 \backslash C_{1}-0.7265469501,0.0874822123,-0.065129249$ $3 \mid C, 0.2662995602,1.0419013144,-0.7987261971 \backslash N, 1.5734039369,0.788137763$ $8,-0.111222541 \backslash C, 2.0131655724,-0.4648273558,0.1594201128 \backslash N, 1.182447970$ $8,-1.5564934845,0.1086065109 \mid N,-1.0433546432,-2.1386859475,-0.44891574$ $06 \mid C_{1},-2.1537226408,-1.3692341269,-0.816824627 \backslash N,-2.0411144687,-0.09878$ $37528,-0.6491621326 \backslash \mathrm{H},-3.0313779549,-1.856951123,-1.2264214153 \backslash \mathrm{N}, 3.270$ $532305,-0.7073759366,0.4754374831 \mathrm{H}, 3.5450274353,-1.6697419599,0.644916$ $5019 \mid \mathrm{H}, 3.9971082196,-0.0027519447,0.4677906567 \backslash \mathrm{H}, 2.2579383898,1.516405$ $6103,-0.2933697605 \backslash H,-0.9064269912,-3.1278373923,-0.630891434 \backslash N,-0.982$ $1605028,0.549187463,1.3971623195 \backslash 0,-1.214382037,1.7463322604,1.5692741$ $83 \backslash 0,-1.0063419008,-0.3097938285,2.2565504524 \backslash 0,0.3986187273,0.6303923$ $576,-2.108537074210,-0.0426446798,2.3707657954,-0.7508952505 \backslash \mathrm{H},-0.1687$ $990567,1.1880892892,-2.6633856221 \mathrm{H},-0.5052297667,2.5560082728,0.091227$ $6047 \backslash$ ।Versi on $=x 86$. Li nux-G03RevB. 05 St at e =1. A Di el ectri $c=78.39 \backslash A 0=4.221$ $\mathrm{HF}=-823.8354463 \backslash \mathrm{RMSD}=6.576 \mathrm{e}-09 \backslash \mathrm{RMSF}=9.546 \mathrm{e}-06 \backslash \mathrm{Di} \mathrm{pol} \mathrm{e}=2.6858962,-1.9122$ $427,-1.0700527 \backslash P G=C 01[X(C 5 H 7 N 604)] \backslash 1 @$

d

$1 \backslash 1 \backslash G|N C-N 3 \backslash F O p t \backslash R B 3 L Y P \backslash 6-31 G(d, p) \backslash C 5 H 7 N 604(1+) \backslash L| U \backslash 07-0 c t-2004 \backslash 0 \backslash \backslash \# B 3$ LYP/ 6-31G** OPT SCRF=(DI POLE, SOLVENT =WATER) ||PCM d | 1, 1|C, 1.1825883124 $-0.8301617612,-0.3030698123 \mid \mathrm{C}, 1.8977907666,0.2802467827,0.15362523931$ $N,-1.5354784032,0.1364186535,-0.02443397031 C_{1}-1.2380657636,-0.88699918$ $18,-0.8582248528 \backslash \mathrm{N}, 0.0901168954,-1.0639480699,-1.1613344575 \backslash \mathrm{N}, 1.895257$ $1808,-1.891500471,\left.0.1958521189\right|^{\prime} \mathrm{C}, 2.9551885981,-1.3981442081,0.90294071$ $93 \backslash \mathrm{N}, 2.9755216578,-0.0850797473,0.8985290562 \backslash \mathrm{H}, 3.6658041396,-2.0463536$ $689,1.3961805237 \backslash \mathrm{N},-2.1353481266,-1.6712766337,-1.4139417286 \backslash \mathrm{H},-3.1260$ $144136,-1.5072083046,-1.25585565161 \mathrm{H},-1.8858545506,-2.5273136759,-1.90$ $067588 \backslash \mathrm{H}, 1.6683837138,-2.8747340849,0.0692848471 \backslash \mathrm{H},-0.8235264232,0.874$ $011434,0.0269848316 \backslash N, 1.6325381618,1.6735918327,-0.071422958310,2.5156$ $747401,2.4878441433,0.161977847210,0.5041688449,1.9821198811,-0.505531$ $3995 \backslash C_{1}-2.7476241514,0.4826700721,0.626421373810,-2.8091761702,1.47131$ $32097,1.30969440910,-3.7122240455,-0.3992042668,0.36895236191 \mathrm{H},-4.5241$ $184526,-0.1746487892,0.8582447527 \backslash \mathrm{H}, 0.2802608955,-1.6534458196,-1.9678$

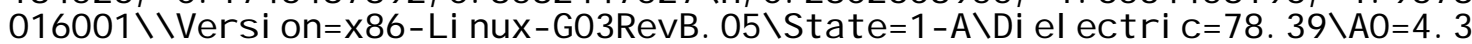
$4 \backslash \mathrm{HF}=-823.8866974 \backslash \mathrm{RMSD}=3.444 \mathrm{e}-09 \backslash \mathrm{RMSF}=4.523 \mathrm{e}-06 \backslash \mathrm{Di} \mathrm{pol} \mathrm{e}=-2.9310509,-6.2$ $754304,-2.0675194 \backslash P G=C 01[X(C 5 H 7 N 604)] 11 @$

e

$1|1| G|N C-N 5 \backslash F 0 p t| R B 3 L Y P \backslash 6-31 G(d, p)|C 5 H 4 N 603 \backslash L| U \backslash 07-0 C t-2004 \backslash 0 \backslash \backslash \# B 3 L Y P \mid$

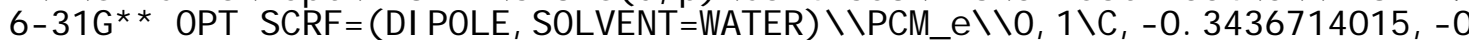
$.8127580945,-0.357292688\left|C_{1}-0.1969267975,0.6588851634,-0.2808408259\right| C_{1}$ - $1.4243516938,1.448809744,0.110431404 \backslash N,-2.5564420168,0.6944855332,0.2$ $725893674 \backslash C_{1}-2.5213814892,-0.6228277295,0.1188688632 \backslash \mathrm{N},-1.4317132767$, $1.4697304045,-0.16835942981 \mathrm{~N}, 0.8936906052,-1.2623274679,-0.6944184581 \mathrm{C}$ $, 1.7756324514,-0.1487654689,-0.8043934951 \backslash \mathrm{N}, 0.9938917518,1.0588385129$, $-0.5513683665 \backslash 0,-1.3624373153,2.6619022569,0.2673742694 \backslash \mathrm{H}, 2.3474363354$ $,-0.0966801624,-1.7320705669 \backslash N,-3.6614208546,-1.3079476859,0.253962446$ $6 \backslash \mathrm{H},-4.5209950889,-0.8091054448,0.4236769449 \backslash \mathrm{H},-3.6659175498,-2.307120$ $2368,0.11082513031 \mathrm{H}, 1.1330458598,-2.2289889391,-0.8663263495 \backslash \mathrm{N}, 2.87535$ $73481,-0.1482447932,0.315283000310,3.9444472002,0.3525619365,-0.004061$ $2393 \backslash 0,2.5651250061,-0.5961745392,1.4053663915 \backslash$ IVersion=x86-Li nux - G03R evB. $05 \backslash$ St at $e=1$ - A $\backslash$ Di el ectri $C=78.39 \backslash A O=4.38 \backslash \mathrm{HF}=-747.0200985 \backslash \mathrm{RMSD}=6.899 \mathrm{e}-$ 
$09 \backslash$ RMSF $=2.145 \mathrm{e}-05 \backslash \mathrm{Di} p 0|\mathrm{e}=-1.1826745,-3.5310893,-1.3278009| P G=C 01 \quad[X(C 5$ H4N603)]11@

f

$1 \backslash 1 \backslash G|N C-N 3 \backslash F O p t \backslash R B 3 L Y P \backslash 6-31 G(d, p) \backslash C 5 H 4 N 603 \backslash L I U \backslash 07-0 c t-2004 \backslash 0 \backslash \backslash \# B 3 L Y P|$ 6-31G** OPT SCRF =(DIPOLE, SOLVENT =WATER) \\PCM $f \backslash \mid 0,1 \backslash C,-0.0300692598,1$. $1987613579,-0.1839553366 \mid C_{1}-0.6891269816,-0.0973919277,0.2341400811 \backslash C_{\text {, }}$ $0.3386229612,-0.9409758787,1.0947721911 \backslash \mathrm{N}, 1.650452043,-0.7185339889,0$. $8100733543 \backslash \mathrm{C}, 2.0066827176,0.3033055608,0.0421460656 \backslash N, 1.221252372,1.39$ $18898448,-0.3635317847 \backslash N,-1.0270421861,2.1326687551,-0.1202695093 \backslash C_{1}-2$ $.1034800067,1.5195284045,0.52832738261 \mathrm{~N},-1.9732969997,0.26496157,0.784$ $9669884 \backslash 0,-0.0966469519,-1.777313224,1.8655130548 \backslash \mathrm{H},-2.9761672315,2.11$ $03270921,0.7844189226 \backslash \mathrm{N}, 3.2847587712,0.4246003453,-0.3224939045 \backslash \mathrm{H}, 3.58$ $91097107,1.2648652053,-0.79295511891 \mathrm{H}, 3.958728222,-0.2474830845,0.0086$ $635906 / \mathrm{H},-0.8807393295,3.1290712579,-0.2283429617 \backslash \mathrm{N},-0.9168464115,-1.0$ $004139665,-1.024790147910,-2.0112307166,-1.5247413978,-1.149303139510$, $0.0451712837,-1.1507395646,-1.7677163789$ । Versi on =x 86-Li nux-G03RevB. 05

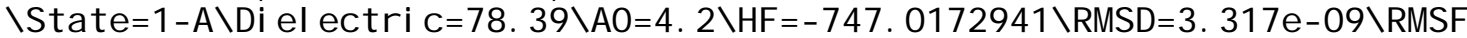
$=2.092 \mathrm{e}-05 \backslash \mathrm{Di}$ pole $=1.3635296,4.0133087,-0.7805117 \backslash \mathrm{PG}=\mathrm{CO} 1$ [X(C5H4N603)] $1 @$

9

$1 \backslash 1 \backslash G \mid N C-N 5 \backslash F 0 p t \backslash R B 3 L Y P \backslash 6-31 G(d, p) \backslash C 5 H 6 N 604 \backslash L I U \backslash 07-0 c t-2004 \backslash 0 \backslash \backslash$ \#B3LYP/ 6. $31 G^{*} *$ OPT $S C R F=(D I P O L E, S O L V E N T=W A T E R) \backslash|P C M g||0,1| C,-0.0427313345,1$. $3010103456,-0.1860870745 \backslash C_{1}-0.7108708464,-0.0430253733,0.0797665305 \backslash C_{\text {, }}$ $0.3117506888,-0.8827255229,0.9206942927 \backslash N, 1.6595420114,-0.7242862884,0$ $.467331355 \backslash \mathrm{C}, 2.0028485958,0.363931586,-0.1424027832 \backslash \mathrm{N}, 1.1990719774,1.5$ $205907717,-0.3453482796 \backslash N,-1.05198935,2.223708407,-0.0183584643 \backslash C_{1}-2.1$ $075548149,1.5472855909,0.59143757251 N,-1.9865906615,0.2704773648,0.698$ $813932 \backslash \mathrm{H},-2.9674844255,2.1079213064,0.9431760764 \backslash \mathrm{N}, 3.2577292677,0.5338$ $420058,-0.62520962881 \mathrm{H}, 3.5437290526,1.4748004773,-0.8481455771 \mathrm{H}, 3.9629$ $874574,-0.1238506906,-0.33217946391 \mathrm{H},-0.8907661177,3.2212409769,0.0311$ $988577 \backslash N,-0.9676021,-0.7870946996,-1.2556746801 \backslash 0,-2.0424909095,-1.346$ $95088,-1.3966972716|0,-0.0490032384,-0.7821791322,-2.0672101062| 0,0.23$ $7545009,-0.4311467655,2.248604945310,-0.0535270012,-2.2355428603,0.809$ $2903825 \backslash \mathrm{H},-0.6730248799,-0.58972942,2.543681924 \backslash \mathrm{H}, 0.7925862862,-2.7033$ $482343,0.8790337164$ । IVersi on =x86. Li nux-G03RevB.05 St at e=1-Al Di el ectric $=78.39 \backslash \mathrm{A} 0=4.23 \backslash \mathrm{HF}=-823.4389308 \backslash \mathrm{RMSD}=7.658 \mathrm{e}-09 \backslash \mathrm{RMSF}=1.267 \mathrm{e}-05 \backslash \mathrm{Di} \mathrm{pol} \mathrm{e}=0$. $6947907,2.7923946,0.7112719 \mid P G=C 01[X(C 5 H 6 N 604)] 11 @$

\section{h}

$1 \backslash 1 \backslash G|N C-N 1 \backslash F 0 p t \backslash R B 3 L Y P \backslash 6-31 G(d, p) \backslash C 5 H 6 N 604 \backslash L I U \backslash 24-N 0 v-2004 \backslash 0 \backslash|$ \#B3LYP/ 6-31G** OPT SCRF=( DI POLE, SOLVENT =WATER) \\Onsager $h \backslash \backslash 0,1 \backslash C, 0.8213367615$ $, 0.2027514749,-0.00001567721 \mathrm{C}, 2.2188491626,0.3528415918,0.0000119791 \mathrm{~N}$, $-1.9364585961,0.2537541804,-0.00000446031 C_{1}-1.3639629852,-0.9207124068$ $,-0.0000409923 \backslash N, 0.0392523832,-0.9189733575,-0.0000703112 \backslash N, 0.34336090$ $32,1.4730556133,0.0000802185 \backslash \mathrm{C}, 1.4276385285,2.3195948074,0.00006247741$ N, 2. $5717508615,1.6830505192,-0.0000093961 \backslash H, 1.3014758724,3.3926456526$, $0.0001082022 \backslash N,-1.9325693305,-2.1367428913,-0.00004161 \backslash H_{1}-2.9454165012$ , - 2. $1820866953,-0.0001163231 \backslash \mathrm{H},-1.3983492255,-2.990918029,-0.000309246$ $1 \backslash H,-0.6869620925,1.5896120418,0.0001961885 \backslash N, 3.1500890107,-0.69835673$ $42,0.000034124 \backslash 0,4.362474647,-0.4492808751,0.0000203695 \backslash 0,2.6869081491$ $,-1.8643370263,0.0000748935 \backslash C,-3.3029561401,0.5120441451,-0.0000222451$ $\mid 0,-3.7358640336,1.6412231581,0.0000003656 \backslash 0,-4.0979508772,-0.59285024$ $62,-0.0000547084 \backslash \mathrm{H},-5.025233405,-0.2969661316,-0.0000656818 \backslash \mathrm{H}, 0.576533$ $6799,-1.7799559048,-0.0000337054 \backslash$ ।Version=x86-Li nux-G98RevA. 7\Di el ectr $i c=78.39 \backslash A 0=4.2 \backslash \mathrm{HF}=-823.5106833 \backslash \mathrm{RMSD}=3.921 \mathrm{e}-09 \backslash \mathrm{RMSF}=6.382 \mathrm{e}-06 \backslash \mathrm{Di} \mathrm{pol} \mathrm{e}=-$ $4.6112785,-1.7509692,-0.00021331 P G=C 01[X(C 5 H 6 N 604)] 11 @$

\section{5-guanidano-4-nitroi mi dazole}

$1 \backslash 1 \backslash G|N C-N 5 \backslash F O p t \backslash R B 3 L Y P \backslash 6-31 G(d, p) \backslash C 4 H 6 N 602 \backslash L| U \backslash 07-0 c t-2004 \backslash 0 \backslash \backslash \# B 3 L Y P \mid$

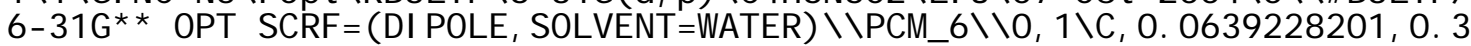


$432084372,0.0307508198 \backslash \mathrm{C},-1.3357697093,0.200027703,0.0083465604 \backslash \mathrm{N}, 2.78$ $1286881,0.9746688627,-0.0718805009 \mid C, 2.4379313299,-0.2607057926,0.0042$ $440714 \backslash N, 1.0645668751,-0.57933265,0.043508404 \backslash N, 0.2695040194,1.6843973$ $172,0.0396332861 \backslash \mathrm{C},-0.9690199215,2.2895663093,0.0228431516 \backslash \mathrm{N},-1.955281$ $7374,1.4302800626,0.0043643035 \backslash H,-1.0685989464,3.3654430282,0.02696984$ $42 \backslash N, 3.2415790927,-1.3737935847,-0.0052805235 \backslash \mathrm{H}, 4.2379551454,-1.209888$ $903,0.0508639127 \backslash \mathrm{H}, 2.9375843641,-2.1963279473,0.4969728919 \mid \mathrm{H}, 1.2484120$ $455,2.0193325859,0.0081439952 \backslash N,-2.0235254275,-1.01963924,-0.029059338$ $810,-3.2628195643,-1.0349921278,-0.0529866152 \mid 0,-1.3263149828,-2.06562$ $11914,-0.0399876021 \backslash \mathrm{H}, 3.7889200839,1.1120624591,-0.0509734174 \backslash \mathrm{H}, 0.7395$ $08646,-1.534359985,-0.0542905196$ I IVersi on =x86- Li nux-G03RevB.05।St at e=1 - A\Di el ectri $C=78.39 \backslash A 0=4.25 \backslash \mathrm{HF}=-634.9337378 \backslash \mathrm{RMSD}=3.599 \mathrm{e}-09 \backslash \mathrm{RMSF}=1.949 \mathrm{e}$

-05\Di pole e=4.9576181,0.4600539,0.4273192।PG=CO1 [X(C4H6N602)]।1@

\section{$8-\mathrm{NO}_{2} \mathrm{GuO}$}

$1 \backslash 1 \backslash G|N C-N 3 \backslash F O p t \backslash R B 3 L Y P \backslash 6-31 G(d, p) \backslash C 5 H 4 N 603 \backslash L| U \backslash 07-0 c t-2004 \backslash 0 \backslash \mid$ \#B3LYP| 6. 31G** OPT SCRF =(DI POLE, SOLVENT =WATER) \|PCM 8||0,1|C, $0.3627672655,-0$. $769907852,0.0123942599|C, 0.2464145271,0.6684124246,-0.005883723| C, 1.49$ $71256346,1.4527067983,-0.0034802894 \backslash N, 2.6295285763,0.6157098966,0.0173$ $002905 \backslash C, 2.6261684268,-0.7742195779,0.033760198 \backslash N, 1.4515419545,-1.4889$ $193912,0.0311497386 \backslash N,-0.9510335933,-1.1952446479,0.0052967763 \mid C_{1}-1.75$ $73521497,-0.0530437617,-0.015765378 \mid N,-1.016694746,1.0823942436,-0.022$ $7142137 \backslash 0,1.5609712829,2.6655192703,-0.0172668369 \backslash N, 3.7628357945,-1.42$ $70781867,0.0519007809 \mid \mathrm{H}, 4.6910228289,-0.9943335334,0.0554420314 \backslash \mathrm{H}, 3.76$ $78824346,-2.4476786381,0.06405545731 \mathrm{H},-1.2708478689,-2.1518049973,0.01$ $37699613 \backslash N,-3.1256421507,-0.1047601049,-0.0276511455 \backslash 0,-3.6925977312$, 1. $2556839369,-0.019104038910,-3.8458351083,0.9615312691,-0.04692865931$ H, 3. $5271419853,1.1044834951,0.0200028356$ II Versi on =x86- Li nux-GO3RevB. 05 |St at e =1-A|Di el ectri $C=78.39 \backslash A 0=3.91 \backslash \mathrm{HF}=-747.1057019 \backslash \mathrm{RMSD}=4.291 \mathrm{e}-09 \backslash \mathrm{RMS}$ $F=3.060$ e $-05 \backslash$ Di pole $=11.2573207,-3.2068304,0.1427105 \backslash P G=C 01$ [X(C5H4N603) ]11@

$\mathrm{H}_{2} \mathrm{O}$

$1 \backslash 1 \backslash G \mid N C-N 3 \backslash F O p t \backslash R B 3 L Y P \backslash 6-31 G(d, p) \backslash H 201 \backslash L I U \backslash 24-N o v-2004 \backslash 0 \backslash \backslash \# B 3 L Y P / 6-31$ G** OPT SCRF =(DI POLE, SOLVENT =WATER) \\PCM $2 \backslash \mid 0,1 \backslash 0,-0.0984756901,0 .,-0$. $0695957369|\mathrm{H},-0.0417682077,0.0 .8948431098| \mathrm{H}, 0.8295737288,0 .,-0.338077$ $2144 \backslash \backslash$ Versi on $=x 86$ - Li nux - G98RevA. 7\St at $e=1-$ Al\ Di el ectri $c=78.39 \backslash A 0=2.48 \backslash$ $\mathrm{HF}=-76.4230104 \backslash \mathrm{RMSD}=1.336 \mathrm{e}-09 \backslash \mathrm{RMSF}=7.769 \mathrm{e}-05 \backslash \mathrm{Di} \mathrm{pol} \mathrm{e}=0.6980275,0 ., 0.493$ $3171 \backslash P G=C 02 V[C 2(01), S G V(H 2)] \backslash 1 @$

$\mathrm{H}_{3} \mathrm{O}^{+}$

$1 \backslash 1 \backslash G \mid N C-N 3 \backslash F O p t \backslash R B 3 L Y P \backslash 6-31 G(d, p) \backslash H 301(1+) \backslash L I U \backslash 24-N O v-2004 \backslash 0 \backslash \backslash$ \#B3LYP/ 6. $31 \mathrm{G} * *$ OPT $S C R F=(D \mid P O L E, S O L V E N T=W A T E R) \backslash \backslash P C M 3 \backslash \backslash 1,1 \backslash 0,0 ., 0 ., 0 . \mid H, 0 ., 0$. $, 0.9764587304 \backslash \mathrm{H}, 0.8456380662,0 .,-0.4882293652 \backslash \mathrm{H},-0.8456380662,0 .,-0.48$ $82293652 \backslash$ Versi on $=x 86$ - Li nux-G98RevA. $7 \backslash$ Di el ectri $C=78.39 \backslash A 0=2.5 \backslash H F=-76.7$ $037356 \backslash R M S D=2.770 \mathrm{e}-09 \backslash \mathrm{RMSF}=1.597 \mathrm{e}-07 \backslash \mathrm{Di} \mathrm{pol} e=0 ., 0 ., 0 . \mid P G=D 03 \mathrm{H}[0(01), 3 \mathrm{C}$ 2(H1)]11@

$\mathrm{NO}_{2}$

$1 \backslash 1 \backslash G \mid N C-N 5 \backslash F O P t \backslash U B 3 L Y P \backslash 6-31 G(d, p) \backslash N 102(2) \backslash L I U \backslash 24-N O V-2004 \backslash 0 \backslash \backslash \# B 3 L Y P / 6$

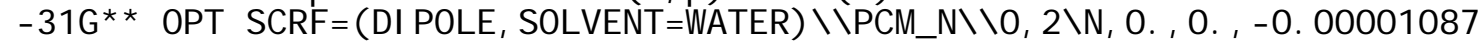
$11|0,0,0 ., 1.2062900378| 0,0 ., 0 .,-1.20628052561 \mid$ Version=x 86 - Li nux - G98Re $\checkmark A .7 \backslash \mathrm{Di}$ electri $C=78.39 \backslash \mathrm{AO}=2.66 \backslash \mathrm{HF}=-205.0140163 \backslash \mathrm{S} 2=0.753584 \backslash \mathrm{S} 2-1=0 . \mid \mathrm{S} 2 \mathrm{~A}=$ $0.750011 \backslash R M S D=5.052 \mathrm{e}-09 \backslash \mathrm{RMSF}=1.690 \mathrm{e}-05 \backslash \mathrm{Di} \mathrm{pol} e=0 ., 0 .,-0.0000594 \backslash P G=C^{*} V$ [C*(01N101)]\।@

\section{a .... $\mathrm{H}_{2} \mathrm{O}(\mathrm{a} \rightarrow 8)$}

$1 \backslash 1|G| N C-N 8 \backslash F 0 p t \backslash R B 3 L Y P \backslash 6-31 G(d, p) \backslash C 5 H 7 N 604(1+) \backslash L I U \backslash 19-N o v-2004 \backslash 0 \backslash \backslash \# B 3$ LYP/ 6-31G** OPT SCRF=(DIPOLE, SOLVENT =WATER) ||gua-b-1||1, $1 \mid C, 0.27427722$ $24,0.5930354956,-0.0932160423 \backslash C, 0.559593507,-0.8453959695,-0.298632944$ $9 \mid C, 1.9718776437,-1.3066262454,-0.1453618786 \backslash N, 2.8348285481,-0.2151989$ $131,0.0712786309 \mid \mathrm{C}, 2.4531201588,1.1115558283,0.1848541672 \backslash \mathrm{N}, 1.15371036$ $23,1.5212216211,0.1456059513 \backslash N,-1.0536458349,0.7129642554,-0.250740050$ 
$8 \backslash C,-1.5774063577,-0.5647587019,-0.6202094595 \backslash N,-0.4800302097,-1.53131$ $13247,-0.599069862 \backslash 0,2.3473788305,-2.4513763735,-0.1954935647 \backslash H,-2.101$ $4859762,-0.5762653783,-1.57828422971 \mathrm{~N}, 3.3724476041,2.0344214452,0.3438$ $272848|\mathrm{H}, 4.3733320723,1.8562720973,0.3592217359| \mathrm{H}, 3.0818355842,3.00778$ $53715,0.4163269115 \backslash \mathrm{H}, 3.8219547761,-0.4586429724,0.1327894106 \backslash \mathrm{H},-1.5610$ $086911,1.6398215973,-0.2938858615 \backslash N,-2.6374443661,-1.1290938182,0.3872$ $821255 \backslash 0,-3.4877388415,-1.8560717933,-0.1132433602 \backslash 0,-2.5117938207,-0$. $8465101171,1.5630402362 \mid 0,-2.3156487989,3.094376413,-0.39486094131 H_{1}-2$ $.2218126527,3.6700174503,0.3791429031 \backslash \mathrm{H},-2.0682298394,3.6597814993,-1$.

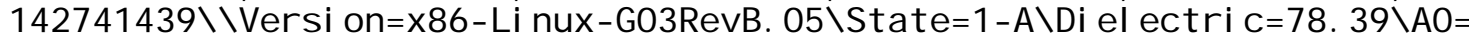
4. $42 \backslash \mathrm{HF}=-823.8646984 \backslash \mathrm{RMSD}=6.591 \mathrm{e}-09 \backslash \mathrm{RMSF}=1.954 \mathrm{e}-06 \backslash \mathrm{Di} \mathrm{pole}=4.8557983,5$. $6189919,-0.5566503 \backslash P G=C 01[X(C 5 H 7 N 604)] \backslash 1 @$

\section{$8 . . . \mathrm{H}_{3} \mathrm{O}$}

$1 \backslash 1 \backslash G \mid N C-N 6 \backslash F O p t \backslash R B 3 L Y P \backslash 6-31 G(d, p) \backslash C 5 H 7 N 604(1+) \backslash L I U \backslash 19-N o v-2004 \backslash 0 \backslash \backslash \# B 3$ LYP/ 6-31G** OPT SCRF=(DI POLE, SOLVENT=WATER) || gua-b-1||1,1|C, 0.83499317 $39,-0.9467482059,-0.0288120181 \backslash C, 0.4298735428,0.4302214621,0.100340391$ $3 \mid C, 1.45903379,1.4736975329,0.1405291344 \backslash N, 2.7458883162,0.8985140265,0$ $.0357978984 \backslash \mathrm{C}, 3.0207323845,-0.4496471382,-0.0920282529 \mid \mathrm{N}, 2.0559922128$, - 1. $4065220215,-0.1173719412 \backslash N,-0.344189464,-1.6429565023,-0.0434002343$ |C, $-1.354397978,-0.6999045451,0.0597805253 \mid N,-0.8832977253,0.560478700$ $2,0.1515797514 \backslash 0,1.2637860083,2.6634296137,0.2416101939 \backslash \mathrm{H},-2.038990270$ $3,2.4016866058,0.1087722116 \backslash N, 4.2751316232,-0.8440235391,-0.1959973481$ IH, 5.0720133146,-0.2159311865,-0.2017156646| H, 4.4652746471,-1.83612042 $29,-0.2991785067 \backslash \mathrm{H}, 3.5117406403,1.5698434431,0.0436991304 \backslash \mathrm{H},-0.4620633$ $195,-2.6450580261,-0.1355888975 \backslash N,-2.6660791125,-1.0279473365,0.048727$ $4278|0,-3.6131322119,-0.0644153835,0.1047803708| 0,-3.0551969835,-2.191$ $582504,0.0065318627 \backslash 0,-2.9697653732,2.4044888095,-0.1800169851 \backslash \mathrm{H},-3.23$ $59871193,0.8865526907,-0.04397535481 \mathrm{H},-2.9430498423,2.7351446836,-1.08$

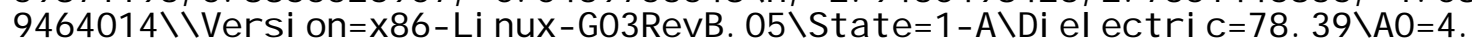
$42 \backslash \mathrm{HF}=-823.8716159 \backslash \mathrm{RMSD}=6.645 \mathrm{e}-09 \backslash \mathrm{RMSF}=1.683 \mathrm{e}-05 \backslash \mathrm{Di} \mathrm{pol} \mathrm{e}=5.435726,-0.48$ $66091,-0.9438536 \backslash P G=C 01[X(C 5 H 7 N 604)] 11 @$

b....H $\mathrm{H}_{2} \mathrm{O}(\mathrm{b} \rightarrow \mathrm{c})$

$1 \backslash 1 \backslash G \mid N C-N 6 \backslash F O p t \backslash R B 3 L Y P \backslash 6-31 G(d, p) \backslash C 5 H 9 N 605(1+) \backslash L I U \backslash 19-N o v-2004 \backslash 0 \backslash \backslash \# B 3$

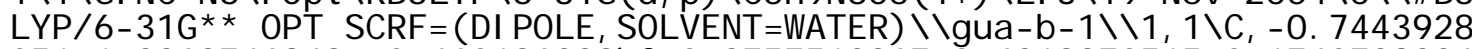
$051,1.2263746849,-0.460196239 \mid C, 0.3757512367,0.4219970515,0.1740728224$ IC, $0.1934378732,-1.0751628105,-0.2937610857 \mid N,-1.2441652525,-1.3627619$ $411,-0.1610953813 \backslash \mathrm{C},-2.2170588951,-0.4626520393,-0.3729493774 \backslash \mathrm{N},-1.963$ $2122387,0.8585331552,-0.6435715447 \backslash N,-0.1472878875,2.3489338659,-0.933$ $3367747 \backslash C, 1.2341137596,2.1636944336,-0.820606548 \backslash N, 1.6003508746,1.0843$ $078034,-0.2235259389|0,0.8273902425,-1.96922471,0.5395244643| \mathrm{H}, 1.91251$ $41123,2.9025117891,-1.2331884877 \backslash N,-3.492667177,-0.8168207985,-0.38133$ $72605 \backslash \mathrm{H},-4.1778554766,-0.1082918027,-0.6140661836 \backslash \mathrm{H},-3.8029382928,-1.7$ $743486499,-0.2937911026 \mid H_{1}-1.4545414092,-2.3543428132,-0.1447484117 \backslash \mathrm{H}$, $-0.6101591004,3.0523953328,-1.49990736381 \mathrm{~N}, 0.2661708486,0.4397247458,1$ $.730004876310,1.3132747888,0.4288204408,2.346582767610,-0.8652707807,0$ $.4143729581,2.193641358810,3.1392139132,-1.2324620873,-0.734952829510$, $0.5244046469,-1.1744910896,-1.6272956561 \mathrm{H}, 1.7805632796,-1.965826387,0$. $305693702 \backslash \mathrm{H}, 3.1682997502,-0.2850595445,-0.5069701625 \backslash \mathrm{H}, 3.9845215599,-1$ $.4804198993,-1.1308863828|\mathrm{H}, 1.502061904,-1.2316678575,-1.6914597166| \mid \mathrm{V}$

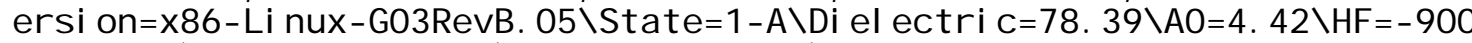
$.2828909 \backslash$ RMSD $=5.962 \mathrm{e}-09 \backslash \mathrm{RMSF}=4.700 \mathrm{e}-05 \backslash \mathrm{Di}$ pole $=-1.2011179,0.5638399,-2$. $9445617 \backslash P G=C 01[X(C 5 H 9 N 605)] \backslash \backslash @$

\section{C.... $\mathrm{H}_{2} \mathrm{O}(\mathrm{b} \rightarrow \mathrm{C})$}

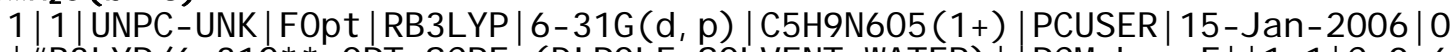
| \#B3LYP/6-31G** OPT SCRF =(DI POLE, SOLVENT =WATER)| PCM b C F || 1, 1|C,0.65 $77789181,0.4123160679,1.0952599529 \mid C, 1.0225266041,0 . \overline{1} 1 \overline{7} 7 \overline{9} 29692,-0.3485$ $882087\left|C_{1}-0.2360995497,-0.3063226793,-1.1315797569\right| N,-1.2533433399,-0$. $811806184,-0.3059851833\left|C_{1}-1.2229773825,-0.7918215805,1.0640078219\right| \mathrm{N}_{1}-$ $0.2672728834,-0.1505427625,1.7915982159 \mid \mathrm{N}, 1.4340674732,1.4663492822,1$. $4335833104|C, 2.00384764,1.9460452555,0.2398514364| N, 1.7891591636,1.237$ 
$8837177,-0.8095684117|0,-0.3102982064,-0.2823397982,-2.3301322685| \mathrm{H}, 2$. $5739864589,2.8679600251,0.2562977079 \mid \mathrm{N},-2.2112023737,-1.3470533359,1.7$ $350789907 \mathrm{H},-2.2477471542,-1.225036781,2.7428279096 \mid \mathrm{H},-2.9835646096,-1$ $.8245132251,1.2860641343|H,-2.1856573934,-0.8369808034,-0.7429160074| H$ , 1. $3146985581,1.9960273083,2.2916202971 \mid N, 1.9749876014,-1.1641118939$, $0.3332834359|0,3.1666560238,-0.9738357277,-0.4795016706| 0,1.4137207464$ , - 2. $2347971963,-0.1653906478 \mid 0,-3.762865147,0.3180435505,-0.9498762686$ | $0,-1.4485529218,2.0015351907,-0.2178905758 \mid \mathrm{H},-4.1561143221,0.41985827$ $42,-1.8296846891|\mathrm{H},-4.5081004561,0.4610071362,-0.3439849683| \mathrm{H},-2.31427$ $06254,1.679952015,-0.5293496728 \mid \mathrm{H},-1.577741285,2.9497859374,-0.0822151$ 394|| Version=x86. Wi n32-G03RevB.05| St at e=1-A|Di elect r i $c=78.39|A 0=4.4| H F$ $=-900.2848624 \mid$ RMSD $=5.989 e-009|R M S F=7.826 e-005| D i p o l e=-5.3083865,1.6612$ $136,3.0154751|P G=C 01[X(C 5 H 9 N 605)]| \mid @$

$\mathrm{C} . . . \mathrm{H}_{2} \mathrm{O}(\mathrm{C} \rightarrow \mathrm{d})$

$1|1| G|N C-N 4 \backslash F O p t| R B 3 L Y P \backslash 6-31 G(d, p)|C 5 H 9 N 605(1+)| L|U \backslash 31-| a n-2005 \backslash 0 \backslash \mid \# B 3$

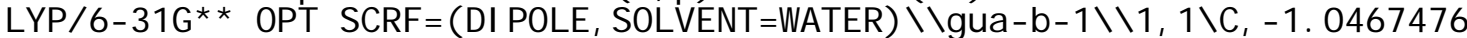
$035,0.157526061,-0.0614029912 \backslash C_{1}-0.9539397658,0.1536899621,1.343488009$ $7 \backslash \mathrm{C}, 2.0540186189,0.0240287321,-0.6844485055 \backslash \mathrm{N}, 1.5500761681,-1.16879133$ $19,-0.7913161628 \backslash \mathrm{C}, 0.2879443587,-1.4203089706,-1.2437934912 \backslash \mathrm{N},-0.79150$ $8453,-0.6732392597,-1.05396503521 N,-1.7176895774,1.3593193221,-0.33473$ $8599 \backslash \mathrm{C},-2.0169820572,2.019419489,0.7890586649 \backslash \mathrm{N},-1.5716737729,1.308468$ $5192,1.8152296678 \backslash \mathrm{H},-2.5314800063,2.9685744414,0.8463252923 \backslash \mathrm{N}, 0.144046$ $4897,-2.5340870962,-1.9733263281 \mathrm{H},-0.7631261632,-2.7637057271,-2.35271$ $89904 \backslash \mathrm{H}, 0.962211324,-3.0182615033,-2.30796652631 \mathrm{H},-1.7929537131,1.7096$ $835702,-1.2937063948 \backslash N,-0.4330992064,-0.7772851025,2.253896745510,-0.5$ $337604498,-0.4927502879,3.462039118210,0.09079797,-1.8033918532,1.8039$ $609692 \backslash 0,1.6704464983,1.1734266891,-1.180227964210,3.1752919562,0.1717$ $012483,0.01617449661 \mathrm{H}, 3.3872752413,-0.704247673,0.374582617210,-0.3848$ $304101,1.8149928773,-2.81998644231 \mathrm{H},-0.6843473488,1.3133863603,-3.5945$ $072915 \backslash \mathrm{H}, 0.9170672701,1.1616246021,-1.8304208365 \mathrm{H},-0.1759100088,2.698$ $3399663,-3.1675132422 \backslash \mathrm{H},-1.6691239543,1.5159495761,2.802371815 \backslash$ Versio

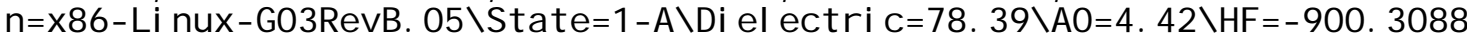
$178 \backslash$ RMSD $=9.464 \mathrm{e}-09 \backslash \mathrm{RMSF}=5.253 \mathrm{e}-06 \backslash \mathrm{Di}$ pole $=-2.3579101,2.9280461,-3.66854$ $76 \backslash P G=C 01[X(C 5 H 9 N 605)] \backslash 1 @$

\section{d.... $\mathrm{H}_{2} \mathrm{O}(\mathrm{c} \rightarrow \mathrm{d})$}

$1 \backslash 1 \backslash G|N C-N 5| F O p t|R B 3 L Y P \backslash 6-31 G(d, p)| C 5 H 9 N 605(1+)|L| U \backslash 25-N 0 V-2004 \backslash 0 \backslash \backslash \# B 3$ $L Y P / 6-31 G * *$ OPT SCRF=(DI POLE, SOLVENT=WATER) ||gua-b-1||1,1|C,-0.3424100 $483,0.3516521575,0.7221851262 \backslash C_{1}-0.3946093319,0.3028497158,2.112587437$ $9 \backslash \mathrm{C}, 0.7542113536,0.2813346697,-2.9187534257 \backslash \mathrm{N}, 1.1742154652,-0.89243244$ $28,-2.1758008682 \backslash \mathrm{C}, 0.942566906,-0.9948826466,-0.8388859988 \backslash \mathrm{N},-0.247586$ $4643,-0.554699078,-0.3478973925 \backslash \mathrm{N},-0.5560488323,1.6724135667,0.4339288$ $206 \backslash \mathrm{C},-0.7199754001,2.3386673375,1.6127657106 \backslash \mathrm{N},-0.6317966161,1.535610$ $9789,2.65000669321 \mathrm{H},-0.8906427283,3.404789126,1.6511746007 \backslash \mathrm{N}, 1.8299371$ $663,-1.5327503648,-0.0232115627 \backslash \mathrm{H}, 1.519816723,-1.7953674591,0.91375450$ $681 \mathrm{H}, 2.7702697564,-1.7531102569,-0.32863654391 \mathrm{H},-0.4940469556,2.036257$ $5297,-0.5167659174 \backslash N,-0.217038395,-0.8379086793,2.9628665448 \backslash 0,-0.5841$ $005749,-0.7602052237,4.13165424810,0.3005342742,-1.8532159732,2.463802$ $075310,0.2423914655,1.2186670117,-2.345475922810,0.9661834523,0.263487$ $3091,-4.2043878111 \backslash \mathrm{H}, 1.3318031406,-0.5356167121,-4.6250757225 \backslash 0,-2.308$ $6010152,0.0075311169,-2.1305772281 / \mathrm{H},-0.9962523206,-0.4199128155,-1.06$ $718993181 \mathrm{H},-2.0658483753,0.7431039194,-2.71256430051 \mathrm{H},-2.8577485913,-0$ $.5735363059,-2.677997346 \backslash \mathrm{H}, 1.8509113938,-1.5260862197,-2.5954609821 \backslash \backslash \mathrm{V}$ ersion=x86-Linux-G98RevA.7\Di el ectri $c=78.39 \backslash A 0=4.42 \backslash H F=-900.3163549 \backslash R M$ $S D=5.321 \mathrm{e}-09 \backslash \mathrm{RMSF}=2.489 \mathrm{e}-04 \backslash \mathrm{Di} \mathrm{pol} e=2.1605205,-1.2431371,-7.0353538 \backslash P G=$ CO1[X(C5H9N605)]।I@

d.... $\mathrm{H}_{2} \mathrm{O}(\mathrm{d} \rightarrow 6)$

$1 \backslash 1 \backslash G|N C-N 1 \backslash F r e q \backslash R B 3 L Y P \backslash 6-31 G(d, p) \backslash C 5 H 9 N 605(1+) \backslash L| U \backslash 18-D e C-2004 \backslash 0 \backslash \backslash \# B 3$ LYP/6-31G** SCRF =(DIPOLE, SOLVENT=WATER) FREQ| I OnSager a $8 \backslash|1,1| C, 1.629$ $5710579,-0.9317620248,-0.109067 \backslash \mathrm{C}, 2.4546770579,0.162452 \overline{9} 752,0.159205 \backslash \mathrm{N}$ 
$,-1.0410699421,0.2391319752,-0.223276 \mid C_{1}-0.7433869421,-0.9182410248,-0$ $.835738 \backslash N, 0.5950560579,-1.2211430248,-1.014257 \backslash N, 2.1519400579,-1.92059$ $00248,0.6886821 \mathrm{C}, 3.2126900579,-1.4012210248,1.373153 \backslash N, 3.4187890579,-0$ $1382850248,1.0730081 \mathrm{H}, 3.7836410579,-1.9910100248,2.076143 \backslash \mathrm{N},-1.650314$ $9421,-1.7478670248,-1.304072 \backslash \mathrm{H},-2.6323339421,-1.4943150248,-1.198117 \backslash \mathrm{H}$ , - 1.4342629421,-2.6923300248,-1.6045121H, 1.7918200579,-2.8679840248, 0 . $769747 \backslash \mathrm{H},-0.2983409421,0.9344469752,-0.195219 \backslash \mathrm{N}, 2.3860530579,1.4832649$ $752,-0.40008810,3.3700550579,2.2146199752,-0.306109 \mid 0,1.3269920579,1.8$ $136769752,-0.958432 \backslash \mathrm{C},-2.3251569421,0.7439069752,0.2440810,-2.32916094$ $21,1.8371439752,0.755835 \backslash 0,-3.2824179421,-0.1078410248,0.015626 \backslash \mathrm{H},-4.2$ $652159421,0.0967219752,0.4056521 \mathrm{H}, 0.7806080579,-1.9792390248,-1.664598$ I H, - 5.8405159421,-0.4398030248,1.559791।0,-5.5850309421,0.1839509752,0 $.8598061 \mathrm{H},-6.2824369421,0.1087069752,0.1875281$ IVersi on =x86-Li nux-G98Re $\checkmark A .7 \backslash \mathrm{Di}$ electri $C=78.39 \backslash \mathrm{AO}=4.2 \backslash \mathrm{HF}=-900.3560234 \backslash \mathrm{RMSD}=9.906 \mathrm{e}-09 \backslash \mathrm{RMSF}=2.002$ e-03। Di pole $=-5.6533261,-7.2305825,-0.725823$ PG=CO1 [X(C5H9N605)]।।@

\section{e....H $\mathrm{H}_{3} \mathrm{O}+(\mathrm{e} \rightarrow \mathrm{a})$}

$1 \backslash 1 \backslash G \mid N C-N 1 \backslash F 0 p t \backslash R B 3 L Y P \backslash 6-31 G(d, p) \backslash C 5 H 7 N 604(1+) \backslash L I U \backslash 22-N O V-2004 \backslash 0 \backslash \backslash \# B 3$ $L Y P / 6-31 G^{* *}$ OPT SCRF =(DI POLE, SOLVENT =WATER) \|gua-b-1\|1,1|C, 0.21046965 $23,-0.8887814037,-0.2328198111 \backslash C, 0.2902035069,0.5872978665,-0.23210529$ $21 \backslash C_{1}-0.9742099134,1.349845137,0.0339451302 \backslash N,-2.074855677,0.486261584$ $7,0.1159215378 \backslash C_{1}-2.0273914087,-0.8940826144,0.0384938741 \backslash N,-0.8500423$ $194,-1.6049270849,-0.0950239471 \backslash N, 1.4907388474,-1.29294986,-0.45234218$ $01 \backslash C, 2.2960483,-0.1416378027,-0.7148653251 \backslash N, 1.4647124032,1.0363758741$ $,-0.4872967796 \backslash 0,-1.0351896637,2.5485239948,0.15356205861 \mathrm{H}, 2.751325948$ $4,-0.1179653797,-1.70806260731 \mathrm{~N},-3.1453109847,-1.5591148001,0.09311909$ $74 \backslash \mathrm{H},-4.0541643209,-1.0439890962,0.1613053809 \backslash \mathrm{H},-3.1275066981,-2.57482$ $27985,0.0299024474 \backslash N, 3.5273829594,-0.0117870028,0.2453322098 \backslash 0,4.51305$ $9317,0.5292901508,-0.247700549910,3.4152208367,-0.4204125439,1.3856122$ $442 \backslash \mathrm{H},-3.0132494181,0.9063659514,0.2164947187 \backslash 0,-4.9912717116,0.418954$ $8254,0.2346997567 \mathrm{H},-5.5880986236,0.4758515358,1.0006104552 \mathrm{YH},-5.58124$ $47004,0.5399135265,-0.5299178771 \mathrm{H}, 1.7392901611,-2.2490632307,-0.673581$ $618 \backslash \backslash$ Versi on $=x 86$ - Li nux-G98RevA. $7 \backslash \mathrm{Di}$ el ectri $C=78.39 \backslash \mathrm{AO}=4.42 \backslash \mathrm{HF}=-823.8746$ $059 \backslash \mathrm{RMS} D=5.783 \mathrm{e}-09 \backslash \mathrm{RMSF}=2.941 \mathrm{e}-06 \backslash \mathrm{Di}$ pole $=-7.5107546,-2.8779753,-0.9157$ $609 \backslash P G=C 01[X(C 5 H 7 N 604)] \backslash 1 @$

\section{a.... $\mathrm{H}_{2} \mathrm{O}(\mathrm{e} \rightarrow \mathrm{a})$}

$1 \backslash 1|G| N C-N 3 \backslash F O p t \backslash R B 3 L Y P \backslash 6-31 G(d, p) \backslash C 5 H 7 N 604(1+) \backslash L I U \backslash 22-N O V-2004 \backslash 0 \backslash \backslash \# B 3$

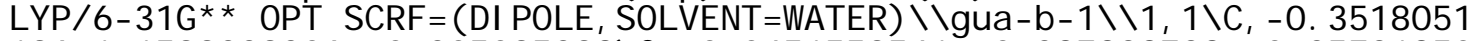
$196,1.1599229206,-0.205095298 \mid C_{1}-0.0451559541,-0.287838738,-0.25731850$ $07 \backslash C, 1.3833236331,-0.6297346637,-0.0282846666 \backslash N, 2.2302043742,0.3538207$ $588,0.1565710425 \backslash \mathrm{C}, 1.8005581668,1.6520543952,0.1340420099 \backslash N, 0.49279024$ $86,2.1115131787,-0.0108024377 \backslash N,-1.6881798644,1.2274042984,-0.44209601$ $21 \backslash C,-2.1751659779,-0.0871907078,-0.7237577609 \backslash N,-1.0659788431,-1.0173$ $517991,-0.5317299319|0,1.7145900537,-1.8691396391,-0.0392713588| H_{1}-2.6$ $24189441,-0.2091891092,-1.7121775618 \backslash \mathrm{N}, 2.7169092639,2.5806643945,0.260$ $1612721 \backslash \mathrm{H}, 3.6993424184,2.3308269651,0.3448645941 \backslash \mathrm{H}, 2.4662545207,3.5647$ $392038,0.2303558436 \backslash N,-3.3185500264,-0.5240357599,0.252806726610,-4.19$ $72343455,-1.2161461442,-0.2489657825 \backslash 0,-3.2437937269,-0.1643388552,1.4$ $134178814 \backslash \mathrm{H}, 4.717822698,-1.9322240902,0.7724145523 \mid 0,4.1336224843,-2.4$ $035163969,0.1591553122 \mid \mathrm{H}, 4.6548329335,-2.5561703412,-0.64417849361 \mathrm{H}, 2$. $7661278956,-2.031000885,0.05511115191 \mathrm{H},-2.1785613089,2.0907618037,-0.6$ $329678238 \backslash$ ।Version=x86-Li nux-G98RevA. $7 \backslash$ Di el ectri $C=78.39 \backslash \mathrm{A} 0=4.42 \backslash \mathrm{HF}=-82$ $3.8557626 \backslash$ RMSD $=8.038 \mathrm{e}-09 \backslash \mathrm{RMSF}=2.322 \mathrm{e}-05 \backslash \mathrm{Di} \mathrm{pol} e=6.1783712,2.7440687,-0$. $9541637 \backslash P G=C 01[X(C 5 H 7 N 604)] \backslash 1 @$

\section{f.... $\mathrm{H}_{2} \mathrm{O}$}

$1 \backslash 1 \backslash G|N C-N 2 \backslash F O p t \backslash R B 3 L Y P \backslash 6-31 G(d, p) \backslash C 5 H 8 N 605 \backslash L I U \backslash 21-N O V-2004 \backslash 0 \backslash \backslash \# B 3 L Y P|$

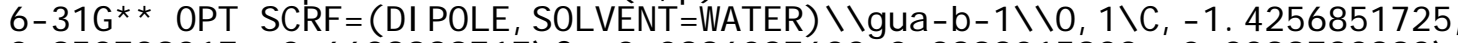
$0.953739017,-0.6628989717 \backslash C_{1}-0.0886087632,0.3898015923,-0.22997828931 \mathrm{C}$ $,-0.0077419552,-1.1305102525,-0.6626476906 \backslash N,-1.182127707,-1.766468965$ $,-0.8061955308 \mid C_{,}-2.3370595554,-1.0885275068,-0.7855980618 \backslash \mathrm{N},-2.503770$ $0288,0.3009560366,-0.8492224177 \backslash N,-1.1588431332,2.2499819641,-1.027679$ 
$1121 \backslash \mathrm{C}, 0.219733903,2.3673923429,-1.0884731974 \backslash \mathrm{N}, 0.9004123345,1.3455202$ $689,-0.6791709877 \backslash \mathrm{H}, 0.662429855,3.2799246267,-1.4717121757 \backslash \mathrm{N},-3.480464$ $1722,-1.753771096,-0.8285247331 \mathrm{H},-4.3537270605,-1.248775425,-0.9302167$ $215 \backslash \mathrm{H},-3.4766845514,-2.7614883924,-0.8952214401 \backslash \mathrm{H},-1.8356606479,2.8642$ $300973,-1.4700855625 \backslash N,-0.0078447509,0.2865570232,1.3306787606 \backslash 0,1.043$ $9264359,0.6335268116,1.857753854110,-0.9731201345,-0.193815719,1.90117$ $43862 \backslash 0,1.1052287918,-1.6541126683,-0.731742356310,4.1146457835,-0.488$ $9426772,2.4458253661 \backslash \mathrm{H}, 2.9080661218,0.6458794748,-0.2909088156 \mid \mathrm{H}, 3.231$ $574441,-0.2674713033,2.7725401774 \backslash 0,3.4580794168,-0.1574899096,-0.2417$ $2243981 \mathrm{H}, 3.9693102392,-0.3904832773,1.47397673531 \mathrm{H}, 2.7692407169,-0.825$ $9402798,-0.4403072691$ । Versi on =x86- Li nux-G03RevB. 05\St at e=1-A Di el ectr $i c=78.39 \backslash A 0=4.42 \backslash \mathrm{HF}=-899.9100819 \backslash \mathrm{RMS} D=3.503 \mathrm{e}-09 \backslash \mathrm{RMSF}=1.431 \mathrm{e}-05 \backslash \mathrm{Di} \mathrm{pol} \mathrm{e}=$ $-7.0840169,2.3879264,-3.114627 \backslash P G=C 01[X(C 5 H 8 N 605)] 11 @$

$g \cdots \mathrm{H}_{2} \mathrm{O}(\mathrm{f} \rightarrow \mathrm{g})$

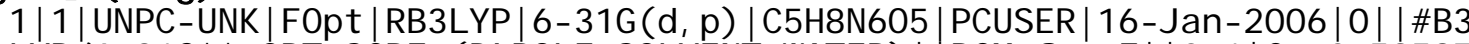
LYP/ 6-31G** OPT SCRF =( DI POLE, SOLVENT=WATER)| PCM $f$ g F| $0,1 \mid C,-0.59587$ $06567,0.133745041,-1.3877061739 \mid C_{1}-0.5962850067,0 . \overline{1} 354608646,0.1367608$ $529|C, 0.9206474403,0.1354276921,0.5326709674| N, 1.547744374,-0.97075640$ $06,-0.1466520383$ |C, 1. $1867203905,-1.2128286953,-1.3689987705 \mid \mathrm{N}, 0.210270$ $1058,-0.5072520061,-2.1360153057 \mid N,-1.5234675195,1.0852949917,-1.73035$ $66798|C,-1.9358371659,1.7052987594,-0.556584451| N,-1.4544853748,1.2380$ $452874,0.5437125425|\mathrm{H},-2.6281487457,2.5391460351,-0.6060540189| \mathrm{N}, 1.756$ $1592217,-2.2140971079,-2.0817075776 \mid \mathrm{H}, 1.5950538143,-2.2363427726,-3.07$ $61774036|\mathrm{H}, 2.5867946274,-2.6460387731,-1.7095310534| \mathrm{H},-1.699578471,1.4$ $041822703,-2.6729159538\left|N_{1}-1.2954928311,-1.1539273743,0.6463996941\right| 0$, 1. $1192405415,-1.4740165968,1.825943953 \mid 0,-2.0326840973,-1.7413245572$, $0.1263203747|0,1.4761147181,1.3352266072,0.1041449249| 0,1.0992528957,0$ $.0404687606,1.9313281742|\mathrm{H}, 1.2592143459,2.0267726858,0.7666204533| \mathrm{H}, 0$. $4686843006,-0.6329777523,2.2476767517 \mid 0,1.3721118166,2.7478668309,2.52$ $30060197|H, 1.1534403203,1.8083833947,2.6736963101| H, 2.3387556345,2.747$ $3328532,2.507344342$ || Version=x86- Wi n32-G03RevB. 05 St at e=1-A| Di el ectric $=78.39|\mathrm{AO}=4.36| \mathrm{HF}=-899.8747225|\mathrm{RMSD}=5.410 \mathrm{e}-009| \mathrm{RMSF}=1.186 \mathrm{e}-004 \mid \mathrm{Di} \mathrm{pol} \mathrm{e}=$ $-0.3361307,0.4771833,-2.3254051|P G=C 01[X(C 5 H 8 N 605)]| \mid @$

$g \cdots \mathrm{H}_{2} \mathrm{O}(\mathrm{g} \rightarrow \mathrm{h})$

$1 \backslash 1 \backslash G \mid N C$ - N6\FOpt $|R B 3 L Y P \backslash 6-31 G(d, p) \backslash C 5 H 8 N 605 \backslash L| U \backslash 22-N O V-2004 \backslash 0 \backslash \backslash \# B 3 L Y P \mid$

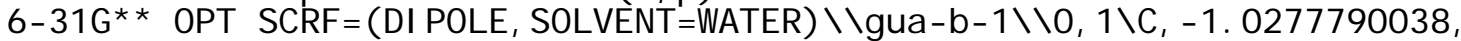
$0.1257953107,-0.0385537399\left|C_{1}-1.1342747367,0.2963337066,1.3631268368\right| C$ , 2. $0819982231,0.1810762283,-0.57460784861 \mathrm{~N}, 1.6409011543,-1.028293884$, $0.4859199245 \backslash \mathrm{C}, 0.4418224457,-1.4423787309,-1.0307904797 \backslash \mathrm{N},-0.730687999$ $6,-0.8746738312,-0.8970473409 \mid N,-1.5390661286,1.3080836045,-0.52288127$ $44 \backslash C_{1},-1.8916193628,2.1025290321,0.5394624315 \backslash N,-1.6748125101,1.5228908$ $191,1.6907398742 \backslash \mathrm{H},-2.3006606634,3.0934146209,0.3978664031 \backslash \mathrm{N}, 0.5207841$ $816,-2.5855798749,-1.74695137361 \mathrm{H},-0.3075904246,-2.9257834801,-2.21064$ $85495 \backslash H, 1.4222200031,-2.9137209235,-2.0559645619 \backslash H,-1.5111788593,1.541$ $2556004,-1.5131431342 \backslash N,-0.8083962622,-0.6449454696,2.361802207510,-1$. $0464991729,-0.3685310874,3.555882374510,-0.3001469415,-1.7245949891,2$. $0085209946 \backslash 0,1.6759196707,1.2087033677,-1.286402782810,3.1587718155,0$. $5207639806,0.1458037997 \backslash \mathrm{H}, 3.415794856,-0.2873735067,0.615116474310,-0$. $2267096961,1.2104121876,-3.1842446529 \mid H_{1}-0.5069001358,0.4109763624,-3$. $6536406709 \backslash \mathrm{H}, 0.9796515878,1.0099158669,-1.9668406985 \backslash \mathrm{H}, 0.0360357911,1$.

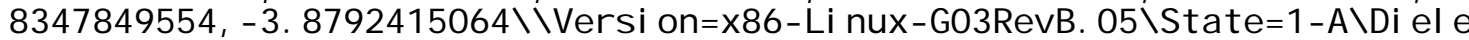
$\mathrm{ctric}=78.39 \backslash \mathrm{A} 0=4.42 \backslash \mathrm{HF}=-899.9241273 \backslash \mathrm{RMSD}=6.575 \mathrm{e}-09 \backslash \mathrm{RMSF}=6.510 \mathrm{e}-06 \backslash \mathrm{Di} \mathrm{po}$ $|e=0.9752782,0.3387057,-6.8519206| P G=C 01[X(C 5 H 8 N 605)] \mid \backslash @$

$h \cdots \mathrm{H}_{2} \mathrm{O}(\mathrm{g} \rightarrow \mathrm{h})$

$1 \backslash 1 \backslash G|N C-N 6 \backslash F 0 p t \backslash R B 3 L Y P \backslash 6-31 G(d, p) \backslash C 5 H 8 N 605 \backslash L I U \backslash 21-N O V-2004 \backslash 0 \backslash \backslash \# B 3 L Y P|$ 6-31G** OPT SCRF=(DIPOLE, SOLVENT =WATER) \|gua-b-1\|0,1|C,-0.6754441226, $-0.4010733124,0.1686290031 \backslash C_{1}-2.0663555285,-0.4144002704,0.148839041 \backslash \mathrm{C}$ $, 2.8574105046,0.0781318017,-0.70988125881 N, 2.1299609791,1.233868036,-0$ $.8712006745 \backslash \mathrm{C}, 0.8845741178,1.2968578334,-0.4766279635 \backslash N, 0.3148925402,0$ 
$5099031446,0.5484093874 \backslash N,-0.3295789639,-1.6542662107,-0.2588100247 \backslash \mathrm{C}$ $,-1.4820365723,-2.3448956495,-0.5060423697 \backslash N,-2.5544018909,-1.62481882$ $31,-0.2630026201 \backslash \mathrm{H},-1.4799403341,-3.3645181264,-0.8641362946 \backslash \mathrm{N}, 0.04095$ $31555,2.1867317971,-1.01370428681 \mathrm{H},-0.8489780324,2.3573484746,-0.55763$ $39425 \backslash \mathrm{H}, 0.370166725,2.7636383425,-1.7722138445 \backslash \mathrm{H}, 0.6535508772,-1.88186$ $2977,-0.4381271366 \backslash N,-2.9465443442,0.6560446955,0.498916137610,-4.1278$ $164987,0.3977508292,0.724831759210,-2.4680757413,1.8044757061,0.565312$ $5704 \backslash 0,2.4375324632,-1.0740019845,-0.5247743245 \backslash 0,4.176397077,0.258666$ $6057,-0.8169383417 \backslash \mathrm{H}, 4.3466679685,1.1980737283,-0.9941706387 \backslash 0,2.09424$ $01955,-1.0729720139,2.18959159221 \mathrm{H}, 1.0210757354,0.0559758341,1.1483048$ $0421 \mathrm{H}, 2.5588060726,-1.3905508448,1.39393308491 \mathrm{H}, 2.7845702977,-0.689418$

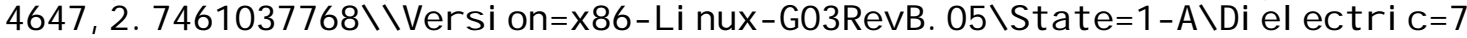

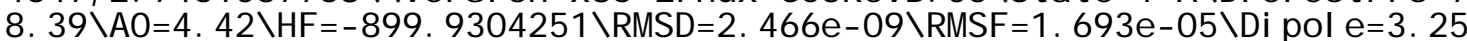
$36936,0.5383454,-0.7136726 \backslash P G=C 01[X(C 5 H 8 N 605)] 11 @$

\section{d.... $\mathrm{H}_{2} \mathrm{O}$}

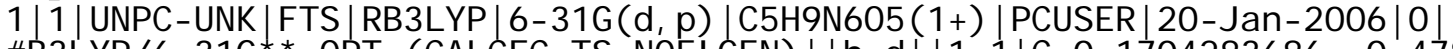
\#B3LYP/ 6-31G** OPT =( CALCFC, TS, NOEI GEN)||h-d||l, 1|C, $0.1704283686,-0.478$ $2668751,1.0019392568|C, 0.1327498895,-0.2479296327,2.3666631368| \mathrm{N}, 0.113$ $0737494,0.1836953333,-1.7790379989 / C_{1}-0.9466416554,-0.4175920687,-1.19$ $94309756|N,-0.9052081039,-0.6255184,0.1376392383| N, 1.5006219886,-0.643$ $1864397,0.7171750607|\mathrm{C}, 2.1832961899,-0.5135825155,1.9162210445| \mathrm{N}, 1.377$ $9451806,-0.2672093652,2.9149157097 \mathrm{H}, 3.2586124002,-0.6057272133,1.9740$ $137544|\mathrm{~N},-2.0652225192,-0.789079436,-1.8268625486| \mathrm{H},-2.1392739025,-0.6$ $857261945,-2.8286187085\left|H_{1}-2.7768214719,-1.3203131178,-1.344907382\right| H_{,} 1$ $.879190113,-0.939235114,-0.1718417754 \mid N,-1.0714005728,-0.0663939791,3$. $1292616564|0,-0.9956221441,0.2223396857,4.3055467665| 0,-2.1400303692$, $0.2292740821,2.4928857158|C, 0.3119731743,0.2983191628,-3.1292407884| 0$, 1. $1584261138,1.080962138,-3.5901824845 \mid 0,-0.417743678,-0.4732177329,-3$ $9425664629|H,-0.1501570001,-0.2841478082,-4.8599890225| H_{1}-1.772497526$ $5,-0.695078123,0.6856193138|\mathrm{H}, 0.9314866956,1.3513359986,-1.2276751483|$ $0,1.5226521986,2.2193302649,-1.4582079031 \mid \mathrm{H}, 2.3772739494,2.2611102195$, - 1.0001892311|H, 1.6112259146, 1.9648167464,-2.4747647228|| Version=x86-W i n32-G03RevB.05 St at $e=1-A|H F=-900.2826064| R M S D=6.664 \mathrm{e}-009 \mid R M S F=9.351 \mathrm{e}-$ $006 \mid$ Di pol e =1.0296335,-0.3525282,-4.2501399|PG=C01 [X(C5H9N605)]||@

\section{$d \cdots \mathrm{H}_{2} \mathrm{O}(\mathrm{d} \rightarrow 6)$}

$1 \backslash 1 \backslash G \mid N C-N 8 \backslash F 0 p t \backslash R B 3 L Y P \backslash 6-31 G(d, p) \backslash C 5 H 9 N 605(1+) \backslash L I U \backslash 21-N o v-2004 \backslash 0 \backslash \backslash \# B 3$ $L Y P / 6-31 G * *$ OPT SCRF=( DI POLE, SOLVENT =WATER) || $\mathrm{gua}-b-1 \mid \backslash 1,1 \backslash \mathrm{C},-1.7300188$ $229,-0.7079594361,0.20348328881 C,-2.4167079577,0.426601007,-0.23390457$ $48 \backslash N, 1.0611395693,0.1439493498,0.2100561357 \backslash \mathrm{C}, 0.6239189318,-0.87127134$ $11,0.972553628 \backslash N,-0.7420277426,-0.9856673708,1.162692075 \backslash N,-2.36330240$ $75,-1.7315875327,-0.4584757213 \mid C_{1}-3.3508345539,-1.193366812,-1.2321252$ $958 \backslash \mathrm{N},-3.4055022298,0.1148220356,-1.1171216373 \backslash \mathrm{H},-3.9849721839,-1.8056$ $197985,-1.8574618469 \backslash \mathrm{N}, 1.4225906978,-1.72699965,1.5725429939 \mid \mathrm{H}, 2.42842$ $21128,-1.6087347188,1.4534043975 \backslash \mathrm{H}, 1.0934372182,-2.5869814785,1.998031$ $2132 \backslash \mathrm{H},-2.1189575146,-2.7166812187,-0.3976459321 \backslash \mathrm{H}, 0.4072584267,0.9109$ $230065,0.0683278803 \backslash N,-2.1929482292,1.7961141583,0.1354553131 \backslash 0,-3.081$ $7218103,2.6175957773,-0.0818487266 \backslash 0,-1.1046198914,2.0753018482,0.6647$ $261725 \backslash C, 2.3987138653,0.4220762639,-0.2955526464 \backslash 0,2.5362035424,1.4232$ $344927,-0.9557456127 \backslash 0,3.2458596429,-0.4954448116,0.0712057974 \backslash \mathrm{H}, 4.247$ $9611523,-0.4658178663,-0.32220647591 \mathrm{H},-1.0202483416,-1.6163284,1.90897$ $83032 \backslash \mathrm{H}, 5.7531334902,-1.3430878101,-1.354835125610,5.5708898504,-0.600$ $3835001,-0.7552527125 \backslash \mathrm{H}, 6.2509985859,-0.6609971911,-0.0640422715 \backslash$ IVers i on $=x 86$. Li nux - G03RevB. $05 \backslash$ St at $e=1$ - A $\backslash$ Di el ect ri $c=78.39 \backslash A 0=4.42 \backslash \mathrm{HF}=-900.34$ $46269 \backslash$ RMSD $=5.259 \mathrm{e}-09 \backslash \mathrm{RMSF}=9.717 \mathrm{e}-06 \backslash \mathrm{Di} p 0 \mid \mathrm{e}=4.4575063,-7.3114732,1.7448$ $499 \backslash P G=C 01[X(C 5 H 9 N 605)] \backslash \backslash @$

\section{G...H $\mathrm{H}_{3} \mathrm{O}^{+}$}

$1 \backslash 1 \backslash G \mid N C-N 8 \backslash F O p t \backslash U B 3 L Y P \backslash 6-31 G(d, p) \backslash C 5 H 7 N 502(1+, 2) \backslash L I U \backslash 21-N O V-2004 \backslash 0 \backslash \backslash \#$ B $3 L Y P / 6-31 G * *$ OPT SCRF=(DI POLE, SOLVENT =WATER) | gua-b-1\|1,2\C,1.310010 $7089,0.6644890182,-0.01687816081 C, 1.1844699475,-0.7628655213,0.0340155$ $104 \backslash C_{1}-0.1635851959,-1.3365809224,0.0472420097 \backslash N,-1.1478004742,-0.3117$ $916143,0.004358581 \backslash \mathrm{C},-0.9008165666,1.0340494831,-0.0423759413 \backslash \mathrm{N}, 0.3605$ 
$011341,1.5644070838,-0.0547263999 \backslash N, 2.669869919,0.8843925486,-0.017317$ $2009 \backslash \mathrm{C}, 3.261168115,-0.3426091905,0.0303076954 \backslash \mathrm{N}, 2.3909190389,-1.357641$ $9572,0.0620638327 \backslash 0,-0.4661163122,-2.5097768041,0.0880405669 \backslash \mathrm{H}, 4.33629$ $54552,-0.4583171647,0.0404886309 \mid N,-1.9109766416,1.8787657125,-0.07799$ $31391 \backslash \mathrm{H},-2.8779005139,1.5293208823,-0.0704987011 \backslash \mathrm{H},-1.7116398074,2.873$ $3470192,-0.1127416966 \backslash \mathrm{H}, 3.1214525395,1.791716454,-0.0474702621 \backslash \mathrm{H},-2.12$ $93000462,-0.6101296574,0.009998190110,-4.051361704,0.0833565332,-0.024$ $6691766 \mathrm{IH},-4.6375511679,0.0420551753,-0.79544553121 \mathrm{H},-4.6466052166,0.0$ $97549841,0.7401318507 \backslash$ IVersion=x86-Li nux-G03RevB.05।St at e =2-AlDi electr i $C=78.39 \backslash A 0=4.3 \backslash \mathrm{HF}=-618.7610492 \backslash S 2=0.764033 \backslash S 2-1=0 . \mid S 2 A=0.750163 \backslash R M S D=$ $7.437 e-09 \backslash$ RMSF $=2.758 e-05 \backslash \mathrm{Di} p 0 \mid e=-2.0387078,3.3990745,-0.1334809 \backslash P G=C 01$ $[X(C 5 H 7 N 502)] \backslash 1 @$

\section{TSa -8}

$1 \backslash 1 \backslash G|N C-N 1 \backslash F T S \backslash R B 3 L Y P \backslash 6-31 G(d, p)| C 5 H 7 N 604(1+) \mid L I U \backslash 04-0 c t-2004 \backslash 0 \backslash \backslash \# B 3 L$ $Y P / 6-31 G * * S C R F=(D \mid P O L E, S O L V E N T=W A T E R) \quad O P T=(R E A D F C, T S, N O E \mid G E N) \quad G E O M=C H$ ECK FREQI।Onsager a $8 \backslash \backslash 1,1 \backslash \mathrm{C}, 0.6904353847,-0.8256133254,0.11466662 \backslash \mathrm{C}, 0$ $.4653432725,0.554 \overline{6} 0 \overline{1} 1271,-0.2601218114 \backslash C_{1}, 1.6366946772,1.4208603293,-0$. $4794193371 \backslash \mathrm{N}, 2.8301530646,0.709531683,-0.1916381107 \backslash \mathrm{C}, 2.9157462525,-0$. $6064827076,0.2142250484 \backslash N, 1.843745731,-1.416579864,0.3376227439 \backslash N,-0.5$ $517136021,-1.3538371723,0.2227756406)^{\prime} C_{1}-1.4735534579,-0.2904407731,0.0$ $790945179 \backslash \mathrm{N},-0.8110747498,0.8474502432,-0.3433517603 \backslash 0,1.6406856895,2$. $5709910754,-0.8454793304 \backslash \mathrm{H},-2.0891138215,0.423774195,1.3396979597 \backslash \mathrm{N}, 4$. $1086954967,-1.1122904131,0.4887728741 \backslash \mathrm{H}, 4.9651230431,-0.5728163607,0.4$ $814839112 \backslash \mathrm{H}, 4.1573614351,-2.0663817009,0.8271554017 \backslash \mathrm{H}, 3.6753599742,1.2$ $701592351,-0.26781488981 \mathrm{H},-0.7648215137,-2.2955091797,0.52901636151 \mathrm{~N},-$ $2.7670995289,-0.5698506116,-0.546039247810,-3.5858202189,0.3738768835$, $-0.575943202510,-3.0050858977,-1.7092819296,-0.9186004612 \backslash 0,-2.8229131$ $876,1.1087911241,1.84779749061 \mathrm{H},-3.362857926,1.3349203371,1.0466062717$ IH, - 2. $3729239289,1.9023212868,2.1939938065$ । Version=x86- Li nux-G03RevB. $05 \backslash \mathrm{St}$ at $e=1-\mathrm{A} \backslash \mathrm{Di}$ el ect $\mathrm{r}$ i $\mathrm{C}=78.39 \backslash \mathrm{AO}=4.43 \backslash \mathrm{HF}=-823.8169488 \backslash \mathrm{RMSD}=4.594 \mathrm{e}-09 \backslash \mathrm{R}$ $M S F=4.539 e-06 \backslash \mathrm{Di}$ pole $=3.5151693,-0.2773787,2.9206923 \backslash \mathrm{PG}=\mathrm{CO}] \quad[\mathrm{X}(\mathrm{C} 5 \mathrm{H} 7 \mathrm{~N} 604$ ) ] $11 @$

\section{$\mathrm{TSB}-\mathrm{C}$}

$1 \backslash 1 \backslash G|N C-N 5 \backslash F T S \backslash R B 3 L Y P \backslash 6-31 G(d, p) \backslash C 5 H 9 N 605(1+)| L \mid U \backslash 04-0 c t-2004 \backslash 0 \backslash \backslash \# B 3 L$ $Y P / 6-31 G^{*} * S C R F=(D \mid P O L E, S O L V E N T=W A T E R) \quad O P T=(R E A D F C, T S, N O E \mid G E N) \quad G E O M=C H$

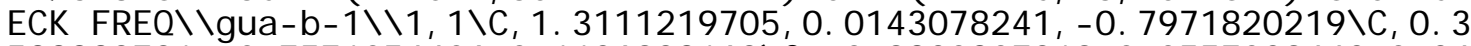
$588883731,-0.7571054406,0.11269381631 C_{1}-0.9820807319,0.0557029442,0.24$ $30911821 \backslash \mathrm{N},-0.6017973292,1.5055071633,0.254320449 \backslash \mathrm{C}, 0.5090842305,2.046$ $371367,-0.2656369561 \backslash N, 1.4441456505,1.2829231393,-0.9292931101 \backslash N, 1.887$ $6751436,-0.9400563878,-1.5818850548 \mid C, 1.1630437651,-2.1192323034,-1.38$ $45417032 \backslash N, 0.2673569914,-2.0833331374,-0.4605234845 \backslash 0,-1.817828624,-0$. $2569896006,1.13545937781 \mathrm{H}, 1.3661183558,-2.9836992512,-2.0075089993 \backslash \mathrm{N}, 0$ $7515517552,3.3447594195,-0.22269348681 \mathrm{H}, 1.5705678003,3.6944585129,-0$. $70774622881 \mathrm{H}, 0.0847605533,4.0248326757,0.1200427591 \backslash \mathrm{H},-1.2462010619,2$. $0775186923,0.7871383817 \backslash \mathrm{H}, 2.4605311455,-0.7241763915,-2.3912507623 \backslash \mathrm{N}, 0$ $.9507263694,-0.8580292663,1.550513339310,0.9147601364,-1.9445483098,2$. $0964641426 \backslash 0,1.3863003084,0.1845630479,2.0202442324 \backslash 0,-3.809569087,0.0$ $059349278,-0.3963856792 \backslash 0,-1.5744398477,-0.114443385,-1.24008806081 \mathrm{H}$, $3.3383986571,-0.0287801635,0.50107226351 \mathrm{H},-4.4063473714,0.7668930452$, $0.4924425451 \backslash \mathrm{H},-2.8464994896,0.0381063097,-1.0085712449 \backslash \mathrm{H},-1.496270075$

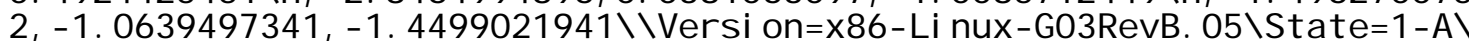
Di electri $c=78.39 \backslash A O=4.35 \backslash \mathrm{HF}=-900.245972 \backslash \mathrm{RMSD}=4.886 \mathrm{e}-09 \backslash \mathrm{RMSF}=4.797 \mathrm{e}-061$ Di pol $e=-1.1297047,3.6980468,-3.5119004 \backslash P G=C 01[X(C 5 H 9 N 605)] \backslash \backslash @$

\section{$\mathrm{TSC} \cdot \mathrm{d}$}

$1 \backslash 1 \backslash G|N C-N 3 \backslash F T S \backslash R B 3 L Y P \backslash 6-31 G(d, p)| C 5 H 9 N 605(1+)|L| U \backslash 12-0 C t-2004 \backslash 0 \backslash \mid \# B 3 L$ $Y P / 6-31 G * * S C R F=(D \mid P O L E, S O L V E N T=W A T E R) \quad O P T=(R E A D F C, T S, N O E I G E N) \quad G E O M=C H$

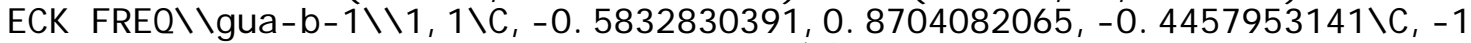
$7272901259,0.1698012496,-0.0245339333 \backslash C_{1} 1.6011025313,-0.6929734423,1$. $3043688136 \backslash N, 1.2843908778,-1.3060317852,0.1007107046 \backslash \mathrm{C}, 1.1794199218,-0$ $.564395579,-1.0940143482 \backslash N, 0.5510161882,0.6005015383,-1.1870103702 \backslash N$, $0.8495969973,2.1588377436,-0.0547574012 \backslash C_{1}-2.0759702204,2.1781557704,0$ 
$5653316157 \backslash \mathrm{N},-2.6274669955,0.9953853848,0.5952718767 \backslash \mathrm{H},-2.4959565409$, $3.0883938528,0.9684602679 \backslash \mathrm{N}, 1.8675723353,-1.0332811583,-2.1235971927 \backslash \mathrm{H}$ $, 1.7327920506,-0.6320939956,-3.0446657876 \mid \mathrm{H}, 2.5724802057,-1.7521415681$ $,-2.0127696581 \backslash \mathrm{H},-0.2372427498,2.9524881076,-0.1976536887 \backslash \mathrm{N},-2.0422348$ $496,-1.2037455673,-0.2350595792 \backslash 0,-3.1161567957,-1.6355431486,0.171896$ $3561 \backslash 0,-1.1963599579,-1.9013772086,-0.8368627345 \backslash 0,1.9381990959,0.4785$ $41782,1.404271365210,1.5242973143,-1.4529175624,2.39159787831 \mathrm{H}, 1.40415$ $0811,-2.3961911571,2.206016194610,2.2709403013,2.2483034205,-0.3946055$ $601 \backslash \mathrm{H}, 1.6135290425,1.6646137029,-1.0179460387 \backslash \mathrm{H}, 2.3698735683,1.6378772$ $706,0.4177515489 \mid \mathrm{H}, 3.1180312905,2.5404692687,-0.7812421192 \backslash \mathrm{H}, 0.9053443$ $402,-2.2471140668,0.0706235722$ I Versi on=x86- Li nux-G03RevB. 05 St at e=1-A $\backslash \mathrm{Di}$ electri $C=78.39 \backslash A 0=4.82 \backslash \mathrm{HF}=-900.2793579 \backslash \mathrm{RMSD}=4.215 \mathrm{e}-09 \backslash \mathrm{RMSF}=1.550 \mathrm{e}-0$ $5 \backslash$ Di pole $=6.0253504,1.4705245,-2.0716754 \backslash P G=C 01[X(C 5 H 9 N 605)] \backslash \backslash @$

\section{$T S d-6$}

$1 \backslash 1 \backslash G \mid N C-N 5 \backslash F T S \backslash R B 3 L Y P \backslash 6-31 G(d, p) \backslash C 5 H 9 N 605(1+) \backslash L I U \backslash 12-N O V-2004 \backslash 0 \backslash \backslash \# B 3 L$ $Y P / 6-31 G * * \quad S C R F=(D I P O L E, S O L V E N T=W A T E R) \quad O P T=(R E A D F C, T S, N O E I G E N) \quad G E O M=C H$

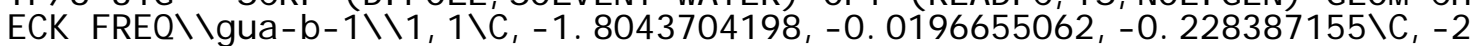
$.2773487955,0.5545052513,0.9536002207 \backslash \mathrm{N}, 1.1073468401,-0.2718979005,-0$. $0499736254 \backslash \mathrm{C}, 0.3581606282,-1.0841808283,-0.7988910358 \backslash \mathrm{N},-1.0122737653$, - 1. $1316722651,-0.5529121055 \backslash N,-2.4592291575,0.6954658123,-1.2033280421$ $|C,-3.2498392057,1.6248054938,-0.595759622| \mathrm{N},-3.1651256327,1.561639367$ $9,0.7162025399 \mid H_{1}-3.8518086488,2.3210161131,-1.162234828 \backslash \mathrm{N}, 0.865879429$ $6,-1.8648009132,-1.72784182891 \mathrm{H}, 1.8797929769,-1.8106518006,-1.86279248$ $77 \backslash \mathrm{H}, 0.3093397182,-2.3447453343,-2.4259579883 \backslash \mathrm{H},-2.3447124528,0.565562$ $4837,-2.2056254001 \backslash \mathrm{H}, 0.6946240707,0.0803452215,0.8055053565 \backslash \mathrm{N},-1.92828$ $89636,0.2109570406,2.305079739210,-2.6883476375,0.5598056665,3.2152000$ $87810,-0.8786972883,-0.4182329277,2.4812804832$ ।C, 2. $5574585862,0.076316$ $4867,-0.193463743410,2.9700599951,0.8250860706,0.662939807910,3.086439$ $033,-0.4971913802,-1.2081693833 \backslash \mathrm{H}, 4.2413005102,-0.2777182127,-1.676776$ $7089 \backslash \mathrm{H},-1.497848242,-1.8904158943,-1.0221170532 \backslash \mathrm{H}, 5.2790005071,0.32867$ $85202,-3.0508789272 \backslash 0,5.2725809766,-0.1380648524,-2.1937100375 \backslash \mathrm{H}, 5.831$ $5049145,-0.9318150898,-2.2926283552$ IIVersi on =x86- Li nux-G03RevB.05।St at $\mathrm{e}=1-\mathrm{A} \backslash \mathrm{Di}$ el ectri $C=78.39 \backslash \mathrm{AO}=4.41 \backslash \mathrm{HF}=-900.3440294 \backslash \mathrm{RMSD}=3.209 \mathrm{e}-09 \backslash \mathrm{RMSF}=5.4$ $36 \mathrm{e}-06 \backslash \mathrm{Dipol} \mathrm{e}=4.4204502,-2.8714552,-8.809788 \backslash \mathrm{PG}=\mathrm{CO} 1$ [X(C5H9N605)]।।@

\section{TSe - a}

$1 \backslash 1 \backslash G \mid N C-N 1 \backslash F T S \backslash R B 3 L Y P \backslash 6-31 G(d, p) \backslash C 5 H 7 N 604(1+) \backslash L I U \backslash 04-0 C t-2004 \backslash 0 \backslash \backslash \# B 3 L$ $Y P / 6-31 G * * \quad S C R F=(D I P O L E, S O L V E N T=W A T E R) \quad O P T=(R E A D F C, T S, N O E \mid G E N) \quad G E O M=C H$

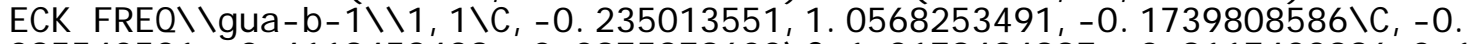
$085540521,-0.4119458438,-0.0975879602 \backslash \mathrm{C}, 1.3179494997,-0.9117423986,0.1$ $553763321 \backslash \mathrm{N}, 2.2761963703,0.0525392867,0.2016240312 \backslash \mathrm{C}, 1.9797210954,1.36$ $25922177,0.1019843226 \backslash \mathrm{N}, 0.6939562528,1.9277690229,-0.0722880888 \backslash \mathrm{N},-1.5$ $768938149,1.2594257563,-0.4239152101 \mid C_{1}-2.1597142912,-0.0164087773,-0$. $6970178679 \backslash \mathrm{N},-1.1799237025,-1.0425704502,-0.349523934 \backslash 0,1.5562609536$, 2. $1206801901,0.2576276561 \mid H_{1}-2.5194205691,-0.1532286704,-1.72061263551$ $\mathrm{N}, 2.9428044913,2.256186589,0.15835718641 \mathrm{H}, 3.919918555,1.9950907692,0.2$ $69602825 \backslash \mathrm{H}, 2.7354715427,3.2451556502,0.0621183631 \backslash \mathrm{N},-3.4631283225,-0.2$ $521971372,0.1445270449|0,-4.4497835001,-0.638248902,-0.4897553699| 0,-3$ $.4337819582,-0.0407715084,1.3431494504 \backslash \mathrm{H}, 3.9602342905,-0.9317002246,0$. $077211153510,4.1645032451$, - 1.8964912272,-0.06943008781 H, 5.0187660741, 2. $2151147512,0.2946722406 \backslash \mathrm{H}, 3.3219102535,-2.3719182759,0.1986382866 \backslash \mathrm{H}$, - 1.8899623838, 2. $1172553695,-0.8584744392$ । IVersi on =x86- Li nux-G03RevB. 05 I St at $e=1-A \mid D i$ elect r i $C=78.39 \backslash A 0=4.29 \backslash \mathrm{HF}=-823.8512377 \backslash$ RMSD $=3.698 \mathrm{e}-09 \backslash \mathrm{RMS}$ $F=4.334$ e-06\Di pole e=9.2333976,1.6073629,-0.5096646\PG=C01 [X(C5H7N604)] $11 @$

\section{TSf - g}

$1 \backslash 1 \backslash G \mid N C-N 5 \backslash F T S \backslash R B 3 L Y P \backslash 6-31 G(d, p) \backslash C 5 H 8 N 605 \backslash L I U \backslash 05-0 C t-2004 \backslash 0 \backslash \backslash \# B 3 L Y P / 6$ $-31 G * * \quad S C R F=(D I P O L E, S O L V E N T=W A T E R) \quad O P T=(R E A D F C, T S, N O E I G E N) \quad G E O M=C H E C K$ FREQ| $\mid$ gua-b- $1 \backslash \mid 0,1 \backslash C,-0.6909893279,0.2470845469,-1.3546684322 \backslash C,-0.586$ $292392,0.2287939601,0.1669202529 \backslash \mathrm{C}, 0.9809125606,0.1491453356,0.3798798$ $989 \backslash N, 1.4497990815,-1.0336668025,-0.2925692729 \mid C, 0.9655726558,-1.25276$ $49108,-1.4724662496 \backslash N,-0.0021658393,-0.4584533152,-2.1615375105 \backslash N,-1.5$ 
$369978226,1.2942275694,-1.6220986685 \backslash C,-1.8007482943,1.9352195538,-0.4$ $132944728 \backslash \mathrm{N},-1.3094173946,1.3933245193,0.64541528231 \mathrm{H},-2.4014060228,2$. $8386051157,-0.4070273317 \backslash \mathrm{N}, 1.3785926997,-2.3108054548,-2.222395618 \backslash \mathrm{H}, 1$ $1800476184,-2.2714615891,-3.21018190341 \mathrm{H}, 2.2244893225,-2.7733793765$,$1.9272288593 \backslash \mathrm{H},-1.7126512928,1.6660170959,-2.54497165 \backslash \mathrm{N},-1.3250654373$, - 1. $0013710318,0.7294812357 \backslash 0,-1.2059368219,-1.2223542769,1.94256286861$ $0,-2.0244955918,-1.6605650648,-0.0198442003 \backslash 0,1.5684020779,1.266644077$ $7,0.0975846006 \backslash 0,1.2085532359,-0.1528135233,1.9405468241 \backslash \mathrm{H}, 1.691087887$ $4,1.9994674346,1.2454322321 \backslash \mathrm{H}, 0.3663648822,-0.5159292793,2.29545400441$ $0,1.7849039801,2.1317186065,2.3304882641 \mathrm{H}, 1.4736749203,0.8989326641,2$. $34903234291 \mathrm{H}, 2.7330294187,2.2290500778,2.496490189$ । IVersion=x86- Li nux-

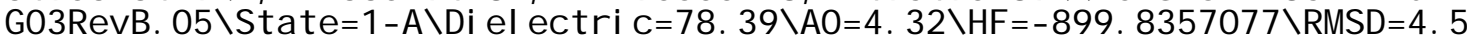
$83 \mathrm{e}-09 \backslash$ RMSF $=1.576 \mathrm{e}-05 \backslash \mathrm{Di}$ pol e $=-0.2432296,0.9895947,-1.6393897 \backslash P G=C 01$ [X ( C5H8N605)] \1@

TSg- $h$

$1|1| G \mid N C-N 5 \backslash F T S \backslash R B 3 L Y P \backslash 6-31 G(d, p) \backslash C 5 H 8 N 605 \backslash L I U \backslash 05-0 C t-2004 \backslash 0 \backslash \backslash \# B 3 L Y P / 6$ - $31 \mathrm{G} * * \quad S C R F=(D \mid P O L E, S O L V E N T=W A T E R) \quad O P T=(R E A D F C, T S, N O E \mid G E N) \quad G E O M=C H E C K$

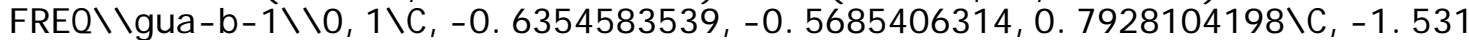
$3017013,-0.6947008441,-0.294518759 \backslash \mathrm{C}, 1.8666868783,0.2971113786,-0.9404$ $490756 \backslash N, 1.2563938699,1.402903153,-0.4881224009 \mid C, 0.6271365174,1.46208$ $67909,0.6799027552 \backslash N, 0.0908232537,0.4068812669,1.3970612161 \backslash N,-0.65289$ $15804,-1.8341796617,1.3382185849 \mid \mathrm{C},-1.5011348613,-2.62627786,0.5938924$ $046 \backslash N,-2.0374204099,-1.9738569039,-0.39621508881 \mathrm{H},-1.6754239324,-3.663$ $1934474,0.8446290445 \backslash N, 0.5748030487,2.6423946738,1.3047399612 \backslash \mathrm{H}, 0.0475$ $3005,2.730540423,2.15908225071 \mathrm{H}, 1.1258709589,3.4148281881,0.9644224963$ $|H,-0.1400114765,-2.1099398931,2.1631660749| N,-1.9812910345,0.32506156$ $25,-1.160102699|0,-2.71779673499,0.026558317,-2.1136012168| 0,-1.6228481$ $463,1.4941556723,-0.9152684704 \backslash 0,2.392077072,-0.6554317924,-0.31241536$ $8 \backslash 0,1.9999672526,0.2569472833,-2.2810179155 \backslash \mathrm{H}, 1.5407826472,1.048417962$ , - 2. $603573159210,2.3262323712,-0.3474949195,2.0872857431 \backslash \mathrm{H}, 1.234059772$ $6,-0.0666854111,2.0488072421 \mathrm{H}, 2.4824226231,-0.5632806611,1.01546926561$ $H, 2.6552239321,-0.9910652844,2.7292511221$ । IVersi on =x86- Li nux-G03RevB. 0 $5 \backslash S t$ at $e=1-A \backslash D i$ el ect ri $c=78.39 \backslash A 0=4.56 \backslash \mathrm{HF}=-899.9005614 \backslash \mathrm{RMSD}=5.347 \mathrm{e}-09 \backslash \mathrm{RM}$ $S F=6.804 e-06 \backslash \mathrm{Di} \mathrm{pole}=2.7209939,0.0172841,4.3661251 \backslash \mathrm{PG}=\mathrm{CO} 1$ [X(C5H8N605)] $11 @$

\section{TSG+- G}

$1 \backslash 1 \backslash G \mid N C-N 3 \backslash F T S \backslash U B 3 L Y P \backslash 6-31 G(d, p) \backslash C 5 H 7 N 502(1+, 2) \backslash L I U \backslash 07-0 c t-2004 \backslash 0 \backslash \backslash \# B$ $3 L Y P / 6-31 G * * \quad S C R F=(D I P O L E, S O L V E N T=W A T E R) \quad O P T=(R E A D F C, T S, N O E I G E N) \quad G E O M=$ CHECK FREOI। guOI।1,2।C, 1.3468458328,0.6494111449,0.0418945192।C,0.8929 $15592,-0.7079798079,-0.0005759131 \mathrm{C},-0.5490842452,-0.9085968353,-0.0371$ $79915 \backslash N,-1.3095147993,0.2395243463,-0.0383950868 \mid C_{1}-0.7361424035,1.464$ $1853057,-0.0060984656 \backslash N, 0.6191008772,1.732754636,0.0369832167 \backslash \mathrm{N}, 2.7224$ $328465,0.5450613524,0.0854585713 \backslash \mathrm{C}, 3.0085716436,-0.786256798,0.0696917$ $513 \backslash N, 1.9258491656,-1.5712121796,0.0139200275 \backslash 0,-1.087929162,-2.041443$ $4646,-0.0618904798 \backslash \mathrm{H}, 4.025714352,-1.1522556617,0.10264793031 \mathrm{~N},-1.50275$ $24164,2.548007029,-0.01342291381 \mathrm{H},-2.5126171927,2.4967957116,-0.041290$ $4901 \backslash \mathrm{H},-1.0647119115,3.4619858331,0.0277344768 \mid \mathrm{H}, 3.3742918423,1.319204$ $8392,0.14198787431 \mathrm{H},-2.7229292066,-0.2782564517,-0.082837186310,-3.359$ $9940473,-1.1500748405,-0.14765123871 H_{1}-2.5241706585,-1.8115947114,-0.0$ $854817517 \backslash \mathrm{H},-3.9566397838,-1.2272574625,0.6153743294 \backslash \backslash$ Versi on $=x 86-$ Li nu $x-G 03 R e v B .05 \backslash$ St at $e=2-A \backslash D i$ el ect $r$ i $C=78.39 \backslash A 0=4.06 \backslash H F=-618.7349653 \backslash S 2=0.7$ $62028 \backslash S 2-1=0 . \mid S 2 A=0.750116 \backslash$ RMSD $=5.157 \mathrm{e}-09 \backslash \mathrm{RMSF}=9.009 \mathrm{e}-06 \backslash \mathrm{Di}$ pol e $=-0.960$ $1812,2.7656558,0.7136887 \backslash P G=C 01[X(C 5 H 7 N 502)] \backslash 1 @$

TSh-d

1| 1 UNPC- UNK|FTS|RB3LYP|6-31G(d, p) | C5H9N605(1+)| PCUSER|08-Feb-2006| 0 | \#B 3 LYP/ 6-31G** OPT =(CALCFC, TS, NOE| GEN)||h-d||l, $1 \mid C, 0.1704283686,-0.478$ $2668751,1.0019392568|C, 0.1327498895,-0.2479296327,2.3666631368| N, 0.113$ $0737494,0.1836953333,-1.7790379989||_{1},-0.9466416554,-0.4175920687,-1.19$ $94309756|N,-0.9052081039,-0.6255184,0.1376392383| N, 1.5006219886,-0.643$ $1864397,0.7171750607|\mathrm{C}, 2.1832961899,-0.5135825155,1.9162210445| \mathrm{N}, 1.377$ $9451806,-0.2672093652,2.9149157097 \mid \mathrm{H}, 3.2586124002,-0.6057272133,1.9740$ 
$137544|\mathrm{~N},-2.0652225192,-0.789079436,-1.8268625486| \mathrm{H},-2.1392739025,-0.6$ $857261945,-2.8286187085\left|H_{1}-2.7768214719,-1.3203131178,-1.344907382\right| H_{1} 1$ $.879190113,-0.939235114,-0.1718417754 \mid N,-1.0714005728,-0.0663939791,3$. $1292616564|0,-0.9956221441,0.2223396857,4.3055467665| 0,-2.1400303692,-$ $0.2292740821,2.4928857158|C, 0.3119731743,0.2983191628,-3.1292407884| 0$, 1. $1584261138,1.080962138,-3.5901824845 \mid 0,-0.417743678,-0.4732177329,-3$ $9425664629|\mathrm{H},-0.1501570001,-0.2841478082,-4.8599890225| \mathrm{H},-1.772497526$ $5,-0.695078123,0.6856193138|\mathrm{H}, 0.9314866956,1.3513359986,-1.2276751483|$ $0,1.5226521986,2.2193302649,-1.4582079031 \mid \mathrm{H}, 2.3772739494,2.2611102195$, $-1.0001892311|\mathrm{H}, 1.6112259146,1.9648167464,-2.4747647228|$ |Version=x86-W in $32-G 03 R e v B .05 \mid S t$ a t $e=1-A|H F=-900.2826064| R M S D=6.664 e-009 \mid R M S F=9.351 e-$ $006 \mid \mathrm{Di}$ pol $e=1.0296335,-0.3525282,-4.2501399 \mid \mathrm{PG}=\mathrm{CO} 1$ [X(C5H9N605)]||@ 